\title{
Coordinate transformation methods to calculate state-to-state reaction probabilities with wave packet treatments
}

\author{
Susana Gómez-Carrasco and Octavio Roncero ${ }^{\text {a) }}$ \\ Instituto de Matemáticas y Física Fundamental, CSIC, Unidad Asociada UAM-CSIC, Serrano 123, \\ 28006 Madrid, Spain
}

(Received 25 April 2006; accepted 5 June 2006; published online 1 August 2006)

\begin{abstract}
A procedure for the transformation from reactant to product Jacobi coordinates is proposed, which is designed for the extraction of state-to-state reaction probabilities using a time-dependent method in a body-fixed frame. The method consists of several steps which involve a negligible extra computational time as compared with the propagation. Several intermediate coordinates are used, in which the efficiency depends on the masses of the atoms involved in the reaction. A detailed study of the relative efficiency of using reactant and product Jacobi coordinates is presented for several systems, and simple arguments are found depending on the masses of the atoms involved in the reaction. It is found that the proposed method is, in general, more efficient than the use of product Jacobi coordinates, specially for nonzero total angular momentum. State-to-state reaction probabilities are obtained for $\mathrm{Li}+\mathrm{FH} \rightarrow \mathrm{LiF}+\mathrm{H}$ and $\mathrm{F}+\mathrm{HO} \rightarrow \mathrm{FH}+\mathrm{O}$ collisions for several total angular momenta. (C) 2006 American Institute of Physics. [DOI: 10.1063/1.2218337]
\end{abstract}

\section{INTRODUCTION}

The most detailed information about reaction dynamics is provided by the state-to-state differential cross section, experimentally obtained in crossed beam apparatus, as recently reviewed. ${ }^{1-3}$ The theoretical simulation of reaction dynamics ${ }^{4}$ is of great interest for the understanding of reaction mechanisms. Up to date, most of the theoretical stateto-state differential cross sections have been obtained using time-independent close coupling methods based on the use of hyperspherical coordinates. ${ }^{5-10}$ However, these methods are somehow limited to triatomic systems and only in few cases have been used to calculate state-to-state probabilities for larger systems as for the case of $\mathrm{H}_{2}+\mathrm{OH}$ collisions ${ }^{11}$ and using reduced dimensionality approaches. Also, the timeindependent algebraic variational methods have been widely used to obtain differential cross sections. ${ }^{12,13}$

In order to study larger systems, the most promising are wave packet methods, as recently reviewed. ${ }^{4,14}$ These methods have allowed the calculation of state-to-state reaction probabilities. ${ }^{16-20}$ However, differential cross sections have been calculated only in few scarce cases using timedependent methods. ${ }^{21}$ The reason is that using timedependent methods is an efficient way to calculate many energies in a single propagation, while the experimental data are obtained for few fixed energies. Therefore, the computational effort is very high specially when all partial waves must be calculated to extract the differential cross section. It is then important to design efficient time-dependent methods to calculate state-to-state magnitudes.

Jacobi coordinates are the most commonly used to study reaction dynamics with wave packet methods, and among them are product Jacobi coordinates to calculate state-tostate reaction probabilities for zero total angular momentum.

${ }^{a)}$ Electronic mail: oroncero@imaff.cfmac.csic.es
This is so because only the initial wave packet needs to be transformed: Since it is separable as a product of one dimensional functions, the transformation is simple and straightforward.

Sometimes, however, it may be desirable to perform a short propagation in the entrance channel using reactant Jacobi coordinates and then transform the wave packet to product Jacobi coordinates to reduce the grids and hence the computational effort. ${ }^{22}$ In such situation it is convenient to design efficient methods to perform the transformation from reactant to product Jacobi coordinates.

The situation becomes more complex for nonzero total angular momentum. Depending on the masses and the dynamics, the body-fixed frame associated to one of them, either reactant or product Jacobi coordinates, may require less number of helicity components to properly describe the wave packet. Typically, the initial wave packet is described in reactant Jacobi coordinate by a single helicity quantum number $\Omega$ (the projection of the total angular momentum in the body-fixed $z$ axis). However, the initial wave packet represented in product Jacobi coordinates needs several $\Omega$ 's. If this number is too large the use of product Jacobi coordinates becomes inefficient and the use of reactant Jacobi coordinates is advisable.

In some particular cases, when the reaction is direct and does not present any wells, the most efficient method is the reactant-product decoupling ${ }^{21,23-26}$ (RPD). In this method, the wave packet is split in reactant and product channels, and its evolution is calculated separately in the best suited coordinates. The flux among the different channels is exchanged using negative imaginary potentials. ${ }^{27}$

When the process is not direct and there are some wells, resonances appear which yield a spreading of the wave packet either in one channel or among all of them. In these cases, the wave packet cannot be split easily without affect- 


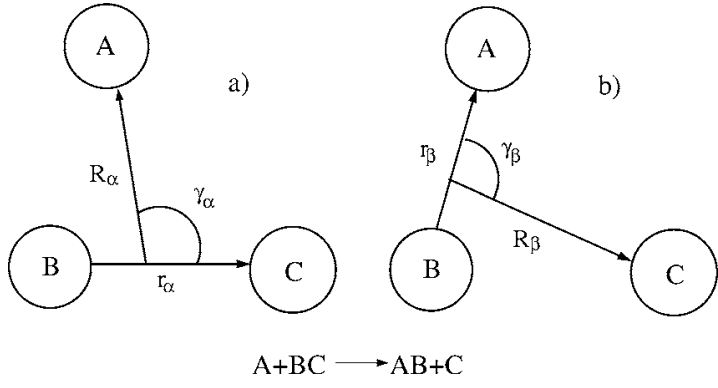

FIG. 1. Jacobi coordinates: (a) Reactant and (b) product.

ing the accuracy of describing the resonances. If the optical potential introduced to transfer the probability from reactant to product coordinates is located in the region where the resonances have a non-negligible amplitude, its description is affected specially when a piece of them may be missing. On the contrary, if to avoid this problem the optical potentials are located far in the product channel, it is more convenient to use a single set of coordinates along the propagation. Bond coordinates have been recently used ${ }^{28}$ to describe reactant and product channels simultaneously in a rather efficient way to propagate the wave packet. The final state distributions are then obtained by transforming from bond to the corresponding Jacobi coordinates.

In this work, the possibility of transforming from reactant to product Jacobi coordinates at each time of the propagation is analyzed. Such procedure has been proposed previously ${ }^{29,30}$ in which the coordinate transformation is done directly in one step. In Sec. II, an efficient method is described to perform such transformation as a sequence of independent steps, so that the computation time required becomes negligible with respect to the evaluation of the action of the Hamiltonian on the wave packet, $H \Psi$. The method is specially well adapted when considering nonzero total angular momentum. The choice of the coordinates strongly depends on the mass combinations of reactants and products. In Sec. III, several reactive systems are compared to analyze the relative efficiency of using a given coordinate set and the transformation procedure as a function of the masses of the atoms involved in the reaction.

Section IV is devoted to discuss the state-to-state probabilities obtained for several cases, such as $\mathrm{Li}+\mathrm{FH} \rightarrow \mathrm{LiF}$ $+\mathrm{H}$ and $\mathrm{F}+\mathrm{HO} \rightarrow \mathrm{FH}+\mathrm{O}$ reactions. Finally, some conclusions are summarized in Sec. V.

\section{COORDINATE TRANSFORMATION}

\section{A. General considerations}

Reactant and product Jacobi coordinates are used in which the vectors $\mathbf{r}_{\alpha}, \mathbf{R}_{\alpha}$ and $\mathbf{r}_{\beta}, \mathbf{R}_{\beta}$ ( $\alpha$ and $\beta$ refer to reactants and products, respectively) are described in Fig. 1. A body-fixed frame is used with the $z$ axis lying along the $\mathbf{R}_{\xi}$ vector $\left(\xi=\alpha\right.$ or $\beta$ ), with $\mathbf{r}_{\xi}$ pointing towards the positive $x$ axis. The angle between the two Jacobi vectors is defined as $\cos \gamma_{\xi}=\mathbf{r}_{\xi} \cdot \mathbf{R}_{\xi} / r_{\xi} R_{\xi}$, with $r_{\xi}$ and $R_{\xi}$ being the module of these vectors, respectively. The body-fixed frame is specified by three Euler angles in each case $\left(\phi_{\xi}, \theta_{\xi}, \chi_{\xi}\right)$.
The wave packet is represented in each set of coordinates as

$$
\left\langle\mathbf{r}_{\xi}, \mathbf{R}_{\xi} \mid \Psi^{J M \epsilon}\right\rangle=\sum_{\Omega_{\xi} \geqslant 0}^{J} \frac{\Phi_{\Omega_{\xi}}^{J M \epsilon}\left(r_{\xi}, R_{\xi}, \gamma_{\xi}\right)}{\left|g_{\xi}\right|^{1 / 4}} W_{M \Omega_{\xi}}^{J \epsilon}\left(\phi_{\xi}, \theta_{\xi}, \chi_{\xi}\right),
$$

where $J$ is the total angular momentum, with projections $M$ and $\Omega_{\xi}$ in the space-fixed and body-fixed $z$ axes, respectively, and $\left|g_{\xi}\right|$ is defined in the Appendix. The $W_{M \Omega_{\xi}}^{J \epsilon}$ are linear combinations of Wigner rotation matrices ${ }^{31}$ with parity $\epsilon$ with respect to total inversion of spatial coordinates, defined as $^{32}$

$$
\begin{aligned}
W_{M \Omega_{\xi}}^{J \epsilon}\left(\phi_{\xi}, \theta_{\xi}, \chi_{\xi}\right)= & \sqrt{\frac{2 J+1}{16\left(1+\delta_{\Omega_{\xi}}\right) \pi^{2}}}\left[D_{M \Omega_{\xi}}^{J *}\left(\phi_{\xi}, \theta_{\xi}, \chi_{\xi}\right)\right. \\
& \left.+\epsilon(-1)^{J+\Omega_{\xi} D_{M-\Omega_{\xi}}^{J *}}\left(\phi_{\xi}, \theta_{\xi}, \chi_{\xi}\right)\right] .
\end{aligned}
$$

A modified Chebyshev propagator is used to propagate the wave packet. ${ }^{33-38}$ The initial real wave packet consists of the product of the rovibrational eigenfunction of the reactant times $G_{v, j, \Omega}\left(R_{\alpha}\right)$, a real linear combination of incoming and outgoing Gaussian functions. ${ }^{38}$ In this approach the evolution operator is expanded in modified Chebyshev polynomials as $^{39}$

$$
\begin{aligned}
\Psi^{J M \epsilon}(t)= & \sum_{k} f_{k}\left(\hat{H}_{s}, t\right) \Psi^{J M \epsilon}(k), \\
& \text { with } f_{k}\left(\hat{H}_{s}, t\right)=\left(2-\delta_{k 0}\right) e^{-i\left(E_{0} t / \hbar\right)}(-i)^{k} J_{k}(t \Delta / \hbar),
\end{aligned}
$$

where $J_{k}(x)$ are Bessel functions of the first kind and $\hat{H}_{s}$ $=\left(\hat{H}-E_{0}\right) / \Delta$ is the scaled Hamiltonian, with $E_{0}=\left(E_{\max }\right.$ $\left.+E_{\min }\right) / 2$ and $\Delta=\left(E_{\max }-E_{\min }\right) / 2 . E_{\max }$ and $E_{\min }$ are the minimum and maximum energy values of the Hamiltonian of the system, $\hat{H}$. The real Chebyshev components of the wave packet, $\Psi^{J M \epsilon}(k)$, are obtained following a modified Chebyshev iterations including a damping function. ${ }^{34}$ Hereafter, the number $k$ will be omitted for simplicity unless it is needed.

Discrete variable representation ${ }^{40-42}$ (DVR) is used, formed by $N$ functions, $G_{i}$, which fulfil approximately the completeness relationship, i.e., $\sum_{i=1}^{N}\left|G_{i}\right\rangle\left\langle G_{i}\right| \approx 1$, with $N=n_{r}$ $+n_{R}+n_{\gamma}$, with $n_{r}, n_{R}$, and $n_{\gamma}$ being the number of functions used to represent each of the three internal coordinates.

In this representation, the wave packet components are expressed by a discrete collection of values in the form

$$
\Phi_{\Omega_{\alpha}}^{J M \epsilon}\left(r_{\alpha}, R_{\alpha}, \gamma_{\alpha}\right)=\sum_{i=1}^{N}\left\langle r_{\alpha}, R_{\alpha}, \gamma_{\alpha} \mid G_{i}\right\rangle\left\langle G_{i} \mid \Phi_{\Omega_{\alpha}}^{J M \epsilon}\right\rangle,
$$

where $\left\langle r_{\alpha}, R_{\alpha}, \gamma_{\alpha} \mid G_{i}\right\rangle \approx \delta\left(r_{\alpha}, R_{\alpha}, \gamma_{\alpha}-r_{\alpha}^{i}, R_{\alpha}^{i}, \gamma_{\alpha}^{i}\right)$ corresponds to approximated Dirac delta functions localized at the grid point $\left(r_{\alpha}^{i}, R_{\alpha}^{i}, \gamma_{\alpha}^{j}\right)$. Thus $\left\langle G_{i} \mid \Phi_{\Omega_{\alpha}}^{J M \epsilon}\right\rangle \equiv\left\langle r_{\alpha}^{i}, R_{\alpha}^{i}, \gamma_{\alpha}^{j} \mid \Phi_{\Omega_{\alpha}}^{J M \epsilon}\right\rangle$ corresponds approximately to the value of the wave packet at a particular grid point.

The wave packet must take a single value independently of the set of coordinates used for the description, i.e.,

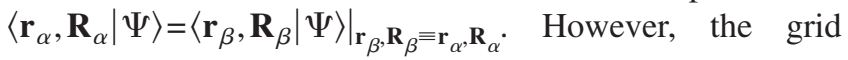


points used in the calculations in reactant and product Jacobi coordinates do not coincide in general. Therefore, to transform a wave packet represented in a grid from reactant to product coordinates, some kind of interpolation must be done. For such purpose, instead of using the $G_{i}$ functions (very localized in a grid point and very oscillating everywhere else), it is better to transform them to a new set of functions $F_{I}$ belonging to the finite basis representation (FBR). These two sets of functions, $\left\{G_{i}\right\}$ and $\left\{F_{I}\right\}$, are related through a unitary transformation and represent the potential and kinetic terms of the Hamiltonian in diagonal matrices, respectively. Such property is used for the propagation of the wave packet, ${ }^{43}$ and it is natural to use the same functions to do the interpolation as

$$
\begin{aligned}
& \left.\Phi_{\Omega_{\alpha}}^{J M \epsilon}\left(r_{\alpha}, R_{\alpha}, \gamma_{\alpha}\right)\right|_{R_{\beta}, X, Y \equiv r_{\alpha}, R_{\alpha}, \gamma_{\alpha}} \\
& =\sum_{I=1}^{N} \sum_{i=1}^{N}\left\langle R_{\beta}, X, Y \mid F_{I}\right\rangle\left\langle F_{I} \mid G_{i}\right\rangle\left\langle G_{i} \mid \Phi_{\Omega_{\xi}}^{J M \epsilon}\right\rangle .
\end{aligned}
$$

For radial coordinates, $G_{i}$ are localized at equidistant grid points and $F_{I}$ are sinus functions, so the transformation between the two sets of functions is performed by fast sinus transforms. The angle $\gamma_{\alpha}$ are described by Gauss-Legendre quadrature points, and the associated FBRs are the spherical harmonic functions.

The direct transformation of a wave packet from reactant to product Jacobi coordinates involves two loops over the grid points describing the three dimensional wave packet in each coordinate set, for $J=0$, which is equivalent to six loops over one dimensional grids. The situation is even more complicated for $J>0$. The simple direct transformation involves more computational effort than the evaluation of the action of the Hamiltonian on the wave packet, becoming the most demanding part of the dynamical calculation. Such procedure has been proposed previously ${ }^{29,30}$ and applied for $J=0$. It is convenient to design efficient methods to do the coordinate transformation as a sequence of steps, each one demanding much lower computational effort, as it is described below.

\section{B. State-to-state probabilities}

The state-to-state probabilities $\left|S_{v j \Omega_{\alpha}, v^{\prime} j^{\prime} \Omega_{\beta}}(E)\right|^{2}$ from an initial state of the reactants $v, j, \Omega_{\alpha}$ towards a $v^{\prime}, j^{\prime}, \Omega_{\beta}$ final state of the products at a given energy $E$ are obtained by a Fourier transform, as described in Refs. 38 and 44. Using the expansion in Eq. (3), the $S$-matrix elements are given by ${ }^{38}$

$$
\begin{aligned}
& S_{v j \Omega_{\alpha}, v^{\prime} j^{\prime} \Omega_{\beta}}(E) \\
& \quad=-i \sqrt{\frac{2 K_{\beta}}{\pi \mu_{\beta}}} \frac{e^{-i k_{\beta} R_{\beta}}}{a_{\alpha}(E)} \sum_{k} c_{k}\left(\hat{H}_{s}, E\right) C_{v^{\prime} j^{\prime} \Omega_{\beta}}(k),
\end{aligned}
$$

where $\mu_{\xi}$ is the reduced mass associated to $R_{\xi}, K_{\xi}$ $=\sqrt{2 \mu_{\xi}\left(E-E_{v_{\xi} j_{\xi}}\right) / \hbar^{2}}$, and

$$
\begin{aligned}
& a_{\alpha}(E)=\frac{1}{2 i} \sqrt{\frac{\mu_{\alpha}}{2 \pi \hbar^{2} K_{\alpha}}} \int d R_{\alpha} e^{i K_{\alpha} R_{\alpha}} G_{\alpha}\left(R_{\alpha}\right), \\
& c_{k}\left(\hat{H}_{s}, E\right)=\int_{0}^{\infty} d t e^{i E t / \hbar} f_{k}\left(H_{s}, t\right) \\
& =\frac{\left(2-\delta_{k 0}\right) \hbar e^{-i k \arccos \left[\left(E-E_{0}\right) / \Delta\right]}}{\sqrt{\Delta^{2}-\left(E-E_{0}\right)^{2}}},
\end{aligned}
$$

with $G_{\alpha}\left(R_{\alpha}\right)$ being the initial Gaussian placed in the entrance channel in state $v j \Omega_{\alpha}$.

In Eq. (6), the $C_{v^{\prime} j^{\prime} \Omega_{\beta}}(k)$ coefficients are given by ${ }^{38,44}$

$$
\begin{aligned}
C_{v^{\prime} j^{\prime} \Omega_{\beta}}(k)= & \int d V_{\beta} \delta\left(R_{\beta}-R_{\beta}^{\infty}\right) \frac{\chi_{v^{\prime} j^{\prime}}\left(r_{\beta}\right) Y_{j^{\prime}, \Omega_{\beta}}\left(\gamma_{\beta}, 0\right)}{\left|g_{\beta}\right|^{1 / 4}} \\
& \times \frac{\Psi^{J M \epsilon}(k)}{\left|g_{\xi}\right|^{1 / 4}} W_{M \Omega_{\beta}}^{J \epsilon}\left(\phi_{\beta}, \theta_{\beta}, \chi_{\beta}\right),
\end{aligned}
$$

where $d V_{\beta}$ and $\left|g_{\beta}\right|$ are defined in the Appendix and $R_{\beta}^{\infty}$ corresponds to an asymptotic distance between the fragments.

It is advantageous to first transform to the single $R_{\beta}^{\infty}$ value, thus reducing a one dimen sional grid to a single point. In this first step, the other two variables, $X$ and $Y$, chosen among $\left(r_{\alpha}, R_{\alpha}, \gamma_{\alpha}\right)$ are kept unchanged. Two possibilities will be considered: (A) $X, Y \equiv R_{\alpha}, \gamma_{\alpha}$ or (B) $X, Y$ $\equiv r_{\alpha}, \gamma_{\alpha}$. The choice of one of these options will depend strongly on the masses of the system, as shown below.

One could perform two more sequential transformation steps to express, finally, the wave packet in product coordinates. However, it is better to transform the product functions $\chi_{v^{\prime} j^{\prime}}\left(r_{\beta}\right) Y_{j^{\prime}, \Omega_{\beta}}\left(\gamma_{\beta}, 0\right)$ to the intermediate $X, Y$ coordinates [denoting them as $\left.\chi_{v^{\prime} j^{\prime} \Omega_{\beta}}(X, Y)\right]$ only once and then evaluate the $C_{v^{\prime} j^{\prime} \Omega_{\beta}}(k)$ at each step as

$$
\begin{aligned}
C_{v^{\prime} j^{\prime} \Omega_{\beta}}(k)= & \int d V^{\prime} \delta\left(R_{\beta}-R_{\beta}^{\infty}\right) \\
& \times \frac{\chi_{v^{\prime} j^{\prime} \Omega_{\beta}}(X, Y)}{\left|g_{\beta}\right|^{1 / 4}} \frac{\Phi_{\Omega_{\beta}}^{J M \epsilon_{1}}\left(R_{\beta}, X, Y\right)}{\left|g^{\prime}\right|^{1 / 4}},
\end{aligned}
$$

where $d V^{\prime}=\sqrt{\left|g^{\prime}\right|} d R_{\beta} d X d Y$ is the volume element in the new intermediate coordinate system, in which the calculation is described in the Appendix.

Therefore, the problem reduces to evaluate this integral in a grid of the intermediate coordinate system $R_{\beta}, X, Y$ as

$$
\begin{aligned}
C_{v^{\prime} j^{\prime} \Omega_{\beta}}(k)= & \sum_{i^{\prime}}\left\langle\Phi_{\Omega_{\beta}}^{J M \epsilon} \mid R_{\beta}^{i^{\prime}}, X^{i^{\prime}}, Y^{i^{\prime}}\right\rangle \\
& \times\left\langle R_{\beta}^{i}, X^{i^{\prime}}, Y^{i^{\prime}} \mid \widetilde{\chi}_{v^{\prime} j^{\prime} \Omega_{\beta}}\right\rangle \delta\left(R_{\beta}^{i^{\prime}}-R_{\beta}^{\infty}\right),
\end{aligned}
$$

where the modified $\tilde{\chi}_{v^{\prime} j^{\prime} \Omega_{\beta}}$ functions are defined as

$$
\begin{aligned}
\left\langle R_{\beta}^{i^{\prime}}, X^{i^{\prime}}, Y^{i^{\prime}} \mid \widetilde{\chi}_{v^{\prime} j^{\prime} \Omega_{\beta}}\right\rangle= & \chi_{v^{\prime} j^{\prime} \Omega_{\beta}}\left(R_{\beta}^{i^{\prime}}, X^{i^{\prime}}, Y^{i^{\prime}}\right) \\
& \times\left[\frac{\left|g^{\prime}\left(R_{\beta}^{i^{\prime}}, X^{i^{\prime}}, Y^{i^{\prime}}\right)\right|}{\left|g_{\beta}\left(R_{\beta}^{i^{\prime}}, r_{\beta}, \gamma_{\beta}\right)\right|}\right]^{1 / 4},
\end{aligned}
$$

where $r_{\beta}, \gamma_{\beta}$ are obtained from $R_{\beta}^{i^{\prime}}, X^{i^{\prime}}, Y^{i^{\prime}}$. These functions are normalized as 


$$
\sum_{i^{\prime}}\left|\left\langle G_{i^{\prime}} \mid \widetilde{\chi}_{v^{\prime} j^{\prime} \Omega_{\alpha}}\right\rangle\right|^{2} \delta\left(R_{\beta}^{i^{\prime}}-R_{\beta}^{\infty}\right)=1 .
$$

Obtaining these functions is simple, because the product functions are products of one dimen sional functions which may be evaluated at the desired point with no additional computational cost and are only needed to be done once.

To calculate $C_{v^{\prime} j^{\prime} \Omega_{\beta}}$ in Eq. (11) it is necessary that the evaluation of $\Phi_{\Omega_{\beta}}^{J M \epsilon}\left(R_{\beta}^{\infty}, X^{i^{\prime}}, Y^{i^{\prime}}\right)$ be done in an efficient way, since it must be done at each iteration. These functions are obtained in two consecutive steps: first the internal coordinate transformation and second the body-fixed transformation.

\section{Partial internal coordinate transformation}

The discretize components in the intermediate coordinates are obtained by interpolation as expressed in Eq. (5) as

$$
\left\langle R_{\beta}^{i^{\prime}}, X^{i^{\prime}}, Y^{i^{\prime}} \mid \Phi_{\Omega_{\alpha}}^{J M \epsilon}\right\rangle=\sum_{i=1}^{N} U_{i^{\prime} i}\left\langle R_{\alpha}^{i}, r_{\alpha}^{i}, \gamma_{\alpha}^{j} \mid \Phi_{\Omega_{\alpha}}^{J M \epsilon}\right\rangle,
$$

where the transformation matrix includes the Jacobian factor for the coordinate transformation as

$$
U_{i^{\prime} i}=\left(\frac{\left|g^{\prime}\right|}{\left|g_{\alpha}\right|}\right)^{1 / 4} \sum_{I=1}^{N}\left\langle R_{\beta}^{i^{\prime}}, X^{i^{\prime}}, Y^{i^{\prime}} \mid F_{I}\right\rangle\left\langle F_{I} \mid R_{\alpha}^{i}, r_{\alpha}^{i}, \gamma_{\alpha}^{i}\right\rangle
$$

The particular expression of the transformation matrix depends on the transformation scheme chosen.

(A) For the $r_{\alpha} \rightarrow R_{\beta}$ transformation, leaving unchanged $X, Y \equiv R_{\alpha}, \gamma_{\alpha}$, it is obtained that

$$
\begin{aligned}
U_{i^{\prime} i}= & \left(\frac{\left|g_{A}^{\prime}\right|}{\left|g_{\alpha}\right|}\right)^{1 / 4} \delta\left(R_{\alpha}^{i^{\prime}}-R_{\alpha}^{i}\right) \delta\left(\gamma_{\alpha}^{i^{\prime}}-\gamma_{\alpha}^{i}\right) \frac{2}{n_{r}+1} \\
& \times \sum_{I=1}^{n_{r}} \sin k^{I} r_{\alpha}^{i} \sin k^{I} r_{\alpha}^{\prime}
\end{aligned}
$$

where $k^{I}=2 \pi\left(n_{r}-1\right) I /\left(2 n_{r}+1\right) \Delta$ is a discretized momentum associated to the discrete sinus transform for a grid composed by $n_{r}$ equidistant points chosen to describe the $r_{\alpha}$ variable in an interval of size $\Delta$ and

$$
\begin{aligned}
r_{\alpha}^{\prime}= & A^{-1}\left[R_{\alpha}^{i^{\prime}} \cos \gamma_{\alpha}^{i^{\prime}}\right. \\
& \left.+\sqrt{\left(R_{\alpha}^{i^{\prime}}\right)^{2}\left(\cos ^{2} \gamma_{\alpha}^{i^{\prime}}-1\right)+\left(\frac{m_{B}+m_{A}}{m_{A}} R_{\beta}^{i^{\prime}}\right)^{2}}\right],
\end{aligned}
$$

with $A=\left(m_{B}+m_{A}\right) / m_{A}-m_{C} /\left(m_{B}+m_{C}\right)$.
(B) For the $R_{\alpha} \rightarrow R_{\beta}$ transformation, leaving unchanged $X, Y \equiv r_{\alpha}, \gamma_{\alpha}$, we get

$$
\begin{aligned}
U_{i^{\prime} i}= & \left(\frac{\left|g_{B}^{\prime}\right|}{\left|g_{\alpha}\right|}\right)^{1 / 4} \delta\left(r_{\alpha}^{i^{\prime}}-r_{\alpha}^{i}\right) \delta\left(\gamma_{\alpha}^{i^{\prime}}-\gamma_{\alpha}^{j}\right) \frac{2}{n_{R}+1} \\
& \times \sum_{I=1}^{N_{R}} \sin K^{I} R_{\alpha}^{i} \sin K^{I} R_{\alpha}^{\prime},
\end{aligned}
$$

where $K^{I}=2 \pi\left(n_{R}-1\right) I /\left(2 n_{R}+1\right) \Delta$ is a discretized momentum associated to the grid formed by $n_{R}$ equidistant points chosen to describe the $R_{\alpha}$ variable in an interval of size $\Delta$ and

$$
\begin{aligned}
R_{\alpha}^{\prime}= & A r_{\alpha}^{i^{\prime}} \cos \gamma_{\alpha}^{j^{\prime}} \\
& +\sqrt{\left(A r_{\alpha}^{i^{\prime}}\right)^{2}\left(\cos ^{2} \gamma_{\alpha}^{i^{\prime}}-1\right)+\left(\frac{m_{B}+m_{A}}{m_{A}} R_{\beta}^{i^{\prime}}\right)^{2}} .
\end{aligned}
$$

\section{Body-fixed transformation: $\left(\phi_{\alpha}, \theta_{\alpha}, \chi_{\alpha}\right)$ $\rightarrow\left(\phi_{\beta}, \theta_{\beta}, \chi_{\beta}\right)$}

The $x-z$ plane is common for the two-body fixed frames, but the $y$ axes are defined with opposite sign to keep $\mathbf{r}_{\alpha}$ and $\mathbf{r}_{\beta}$ along the positive $x$ axes, as defined in Fig. 1. The transformation between the two body-fixed frames can be accomplished by a rotation of $(0, \varphi, \pi)$ Euler angles, where $\cos \varphi$ $=\mathbf{R}_{\alpha} \cdot \mathbf{R}_{\beta} / R_{\alpha} R_{\beta}$. Thus the transformation between the Wigner matrices used in the expansion of the wave packet is performed as ${ }^{31}$

$$
\begin{aligned}
& D_{M \Omega_{\alpha}}^{J *}\left(\phi_{\alpha}, \theta_{\alpha}, \chi_{\alpha}\right)=\sum_{\Omega_{\beta}} D_{M \Omega_{\beta}}^{J *}\left(\phi_{\beta}, \theta_{\beta}, \chi_{\beta}\right) D_{\Omega_{\beta} \Omega_{\alpha}}^{J *}(0, \varphi, \pi), \\
& D_{M \Omega_{\beta}}^{J^{*}}\left(\phi_{\beta}, \theta_{\beta}, \chi_{\beta}\right)=\sum_{\Omega_{\alpha}} D_{M \Omega_{\alpha}}^{J^{*}}\left(\phi_{\alpha}, \theta_{\alpha}, \chi_{\alpha}\right) D_{\Omega_{\alpha} \Omega_{\beta}}^{J^{*}}(0, \varphi, \pi) .
\end{aligned}
$$

Introducing these expressions in Eqs. (1) and (2), the wave packet components in product coordinates can be obtained according to

$$
\left\langle R_{\beta}^{i^{\prime}}, X^{i^{\prime}}, Y^{i^{\prime}} \mid \Phi_{\Omega_{\beta}}^{J M \epsilon}\right\rangle=\sum_{\Omega_{\alpha} \geqslant 0} T_{\Omega_{\beta^{\prime}} \Omega_{\alpha}}\left(\varphi^{i^{\prime}}\right)\left\langle R_{\beta}^{i^{\prime}}, X^{i^{\prime}}, Y^{i^{\prime}}\right| \Phi_{\Omega_{\alpha}}^{J M \epsilon},
$$

where the transformation matrix is given by

$$
T_{\Omega_{\beta} \Omega_{\alpha}}\left(\varphi^{i^{\prime}}\right)=\sqrt{1+\delta_{\Omega_{\beta} 0}} \sum_{\Omega_{\alpha} \geqslant 0}^{J}\left\{\frac{D_{\Omega_{\alpha} \Omega_{\beta}}^{J}\left(0, \varphi^{i^{\prime}}, \pi\right)+\epsilon(-1)^{J+\Omega_{\alpha}} D_{-\Omega_{\alpha} \Omega_{\beta}}^{J}\left(0, \varphi^{i^{\prime}}, \pi\right)}{\sqrt{1+\delta_{\Omega_{\alpha} 0}}}\right\} .
$$


The angle $\varphi$ is calculated at each grid point according to

$$
\cos \varphi=\frac{A r_{\alpha} \cos \gamma_{\alpha}-R_{\alpha}}{\sqrt{\left(A r_{\alpha}\right)^{2}+R_{\alpha}^{2}-2 A r_{\alpha} R_{\alpha} \cos \gamma_{\alpha}}} .
$$

In the intermediate coordinates, the values of $r_{\alpha}$ or $R_{\alpha}$ are calculated using Eqs. (17) or (19) depending on the option chosen, (A) or (B), respectively.

In Eq. (9) it has been assumed, for simplicity, that $R_{\beta}^{\infty}$ is sufficiently large so that the Coriolis coupling is already zero. However, this fact would in general require to integrate up to very long distances thus increasing the computational effort. The integration can be performed in a shorter interval by using body-fixed Bessel functions, ${ }^{28,30,45-47}$ which satisfy the asymptotic behavior for long distances where the potential vanishes but the rotational barrier and Coriolis couplings are still important.

\section{E. Summary}

In summary, the method consists on the following steps.

(1) Calculation of the modified product wave functions $\left\langle G_{i^{\prime}} \mid \widetilde{\chi}_{v^{\prime} j^{\prime} \Omega_{\beta}}\right\rangle$ in Eq. (12) in a given grid prior to the evolution of the wave packet. Thus, their normalization can be easily converged, checking the adequacy of the transformation scheme chosen [option (A) or (B)] and reducing the integration intervals as much as possible to save storage requirements and computer time.

(2) Calculation of the transformation matrix $U_{i^{\prime} i}$ given by Eq. (16) or (18), depending on the option chosen [(A) or (B)], and the body-fixed transformation matrix $T_{\Omega_{\beta} \Omega_{\alpha}}\left(\varphi^{i^{\prime}}\right)$ given by Eq. (23) prior to the propagation in time. The storage requirements of these two matrices are smaller than that of one wave packet.

(3) At each iteration, the wave packet is, first, transformed to the intermediate coordinates as given in Eq. (14) and, second, transformed to the products body-fixed frame, Eq. (22). The number of operations required is equivalent to those required to evaluate a norm and negligible as compared to the calculation of $H \Psi$.

(4) At each iteration, the $C_{v^{\prime} j^{\prime} \Omega_{\beta}}(k)$ is evaluated by evaluating the integral in Eq. (11). Again, the computer time is negligible with respect to that associated to $H \Psi$, provided that the number of product states is small and the integration intervals are properly adapted.

(5) After the propagation, the desired $S$-matrix elements are calculated using Eq. (6).

\section{MASS COMBINATION EFFECTS ON COORDINATE TRANSFORMATION}

A priori, the most efficient way to calculate state-to-state reaction probabilities is using product Jacobi coordinates to avoid the computational cost of coordinate transformation at each iteration. However, using efficient methods as those described in the previous section, the transformation does not involve a significant additional computational effort as compared with the wave packet propagation. Therefore, the efficiency of using reactant or product Jacobi coordinates will
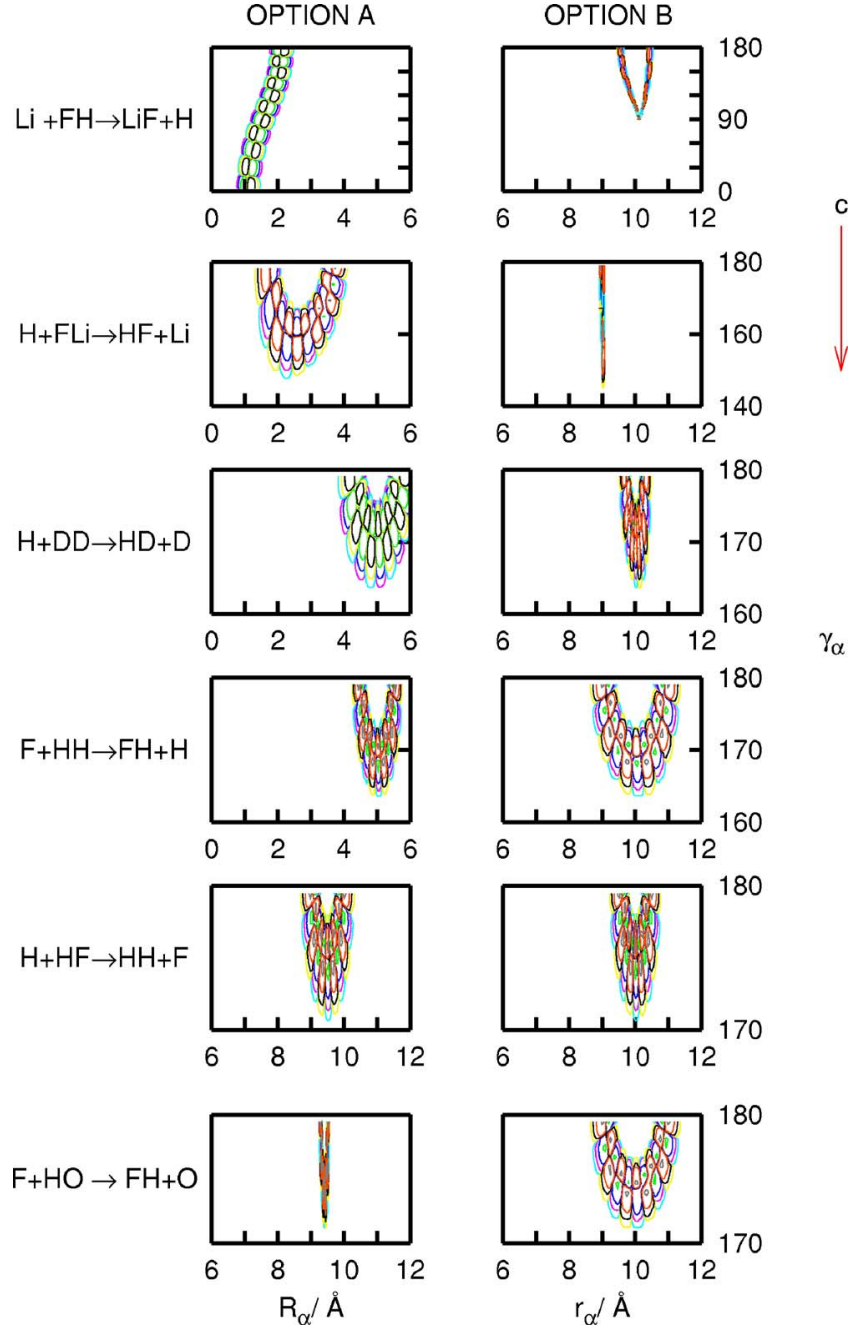

FIG. 2. Normalized product wave functions expressed in either $\left(R_{\alpha}, \gamma_{\alpha}\right)$ [option (A)] or $\left(r_{\alpha}, \gamma_{\alpha}\right)$ [option (B)] at $R_{\beta}^{\infty}=10 \AA$ for the state $v^{\prime}=1, j^{\prime}$ $=10$ of the corresponding products for the following reactions, presented in increasing order of the mass factor $c$.

depend on the number of functions, either in the DVR or FBR representations, needed to converge the calculations. Two major aspects will affect the convergency: (a) the description of final product states using reactant Jacobi coordinates or the description of the initial state of reactants when using product Jacobi coordinates (this aspect will be divided in two parts, internal coordinates and helicity components) (b) and the convergency of the wave packet propagation, which depends on the details of the potential energy surfaces (PESs) and energy interval considered, as will be studied separately below.

To analyze how different coordinate sets describe on $A$ $+B C \leftrightarrow A B+C$ reaction, it is convenient to study the effect of the mass combinations. Traditionally, this is done using the skew angle $\beta$ defined as ${ }^{48}$

$$
\cos ^{2} \beta=a c \quad \text { with }\left\{\begin{array}{l}
c=m_{C} /\left(m_{B}+m_{C}\right) \\
a=m_{A} /\left(m_{A}+m_{B}\right) .
\end{array}\right.
$$

Since $\beta$ is symmetric for direct and reverse reactions, it cannot be used directly to decide which set of coordinates will be the most suitable. Therefore, for such purpose, we shall 


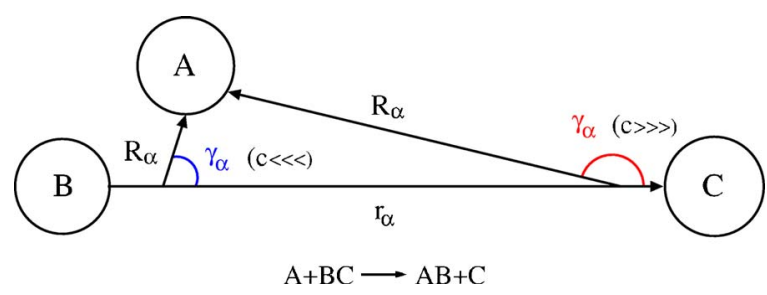

FIG. 3. Reactant Jacobi coordinates for the description of products for different mass coefficients $c$.

use the $a$ and $c$ mass factors in order to describe the important differences in the description of the two processes. These two factors are defined in the $[0,1]$ interval, and they interchange their role for the reverse reactions. Also, mass factor $A$, used in Eq. (17), can be redefined as $A=a^{-1}-c$.

Several systems with different mass combinations (heavy $\equiv H$, light $\equiv L$ ) will be compared:

(1) $H+H^{\prime} L \leftrightarrow H H^{\prime}+L$, applied to $\mathrm{Li}+\mathrm{FH} \leftrightarrow \mathrm{LiF}+\mathrm{H}$, using the PES of Ref. 49, which has the mass factors $a$ $=0.27$ and $c=0.05$ for the direct process,

(2) $L^{\prime}+L L \leftrightarrow L^{\prime} L+L$, in the prototypical $\mathrm{H}+\mathrm{D}_{2} \leftrightarrow \mathrm{HD}+\mathrm{D}$, using the PES of Refs. 50 and 51 which has the mass factors $a=1 / 3$ and $c=1 / 2$ for the direct reaction,

(3) $H+L L \leftrightarrow H L+L$, applied to the prototype $\mathrm{F}+\mathrm{H}_{2} \leftrightarrow \mathrm{FH}$ $+\mathrm{H}$, using the PES of Ref. 52, with mass factors $a$ $=0.95, c=0.5$ for direct reaction, and

(4) $H+L H^{\prime} \leftrightarrow H L+H^{\prime}$, applied to $\mathrm{F}+\mathrm{HO} \leftrightarrow \mathrm{FH}+\mathrm{O}$ reaction for the $1^{3} A^{\prime \prime}$ state of Ref. 53, with $a=0.95, c$ $=0.94$ for the direct process.

Product wave functions for the rovibrational state $\left(v^{\prime}, j^{\prime}\right)=(1,10)$ are shown in Fig. 2 expressed in the intermediate coordinates $\left(R_{\alpha}, \gamma_{\alpha}\right)$ or $\left(r_{\alpha}, \gamma_{\alpha}\right)$ for options (A) and (B), respectively, and ordered with respect to the mass factor c.

For $c \rightarrow 0$, as it is the case of $\mathrm{Li}+\mathrm{FH} \rightarrow \mathrm{LiF}+\mathrm{H}$ reaction, option (A) represents the $\operatorname{LiF}\left(v^{\prime}=1, j^{\prime}=10\right)$ product functions nearly as a product of a function in $\gamma_{\alpha}$ times another one in $R_{\alpha}$, with a rather small curvature, as shown in the corresponding panel of Fig. 2. When $c$ increases, the product wave functions are restricted to a progressively smaller $\gamma_{\alpha}$ interval, as in the rest of the cases in Fig. 2. When $c$ increases, the center of mass of the $B C$ molecule shifts toward the scaping atom $C$, and as products fly apart, $\gamma_{\alpha}$ tends to be restricted about $\pi$, as shown graphically in Fig. 3 .

As product functions are gradually restricted to narrower angular intervals, the number of angular grid points necessary for its proper representation also increases. In order to quantify this fact, the number of angular grid points needed to converge the norm of the product state $\left(v^{\prime}, j^{\prime}\right)=(1,10)$ at $R_{\beta}=10 \AA$ using reactant Jacobi coordinates is shown in Table I. In this table, integration in the $[0.25,14] \AA$ radial intervals has been considered and the density of radial and angular grids was determined to obtain the norm of the $A B\left(v^{\prime}=1, j^{\prime}=10\right)$ products with an error smaller than $0.1 \%$.

Moreover, the representation of the initial state of reactants in terms of product Jacobi coordinates is affected by the same kind of arguments but reversed: When $a$ is very small, the reactants are very well described by using product Jacobi coordinates. As an example, for the $\mathrm{H}+\mathrm{FLi} \rightarrow \mathrm{HF}+\mathrm{Li}$ reaction with small $a$, the $\operatorname{LiF}$ reactants present an uniform distribution over the whole $\gamma_{\beta}$ coordinate, while for the $\mathrm{F}+\mathrm{H}_{2}$ or $\mathrm{F}+\mathrm{HO}$ reactions with $a \approx 1$, the angular interval is very small (see Fig. 2).

Therefore, since small $c$ favors the description of products with reactant coordinates while small $a$ favors the description of the initial state using product coordinates, the relative value of these two mass factors may be used to decide which coordinate set to use: For describing the $A+B C$ $\rightarrow A B+C$ state-to-state reaction probabilities, it is convenient to use reactant Jacobi coordinates if $a \gg c$, product Jacobi coordinates if $a \ll c$, and if $a \approx c$ both cases should be compared in efficiency. This finding is corroborated by determining the number of angular grid points needed to converge the norm of either reactant or product states, as listed in Table I.

Once reactant Jacobi coordinates have been chosen, now it is important to decide whether using option (A) or (B), since they can yield a significant reduction in the number of points of radial grids. For $\mathrm{Li}+\mathrm{FH} \leftrightarrow \mathrm{LiF}+\mathrm{H}$ reaction of minimum $\cos \beta$, option (B) yields to a wave function of $\mathrm{LiF}$ products located in a rather narrower ring than option (A), as a function of $r_{\alpha}$ and $R_{\alpha}$, respectively (see Fig. 2). In such situation, option (A) is advisable since $r_{\beta} \approx R_{\alpha}$ and less dense radial grids are needed, as can be seen in Table I.

For intermediate $\cos \beta$ factors, as it is the case of $\mathrm{H}$ $+\mathrm{D}_{2}$ and $\mathrm{F}+\mathrm{H}_{2}$, the radial intervals or, more clearly, the number of radial grid points should be analyzed in more

TABLE I. Mass factors and number of radial $\left(n_{r}, n_{R}\right)$ and angular points $\left(n_{\gamma}\right)$ needed to converge the (1) norm of the $A B\left(v^{\prime}=1, j^{\prime}=10\right)$ product state in the reactant intermediate Jacobi coordinates for option (A) or (B) and (2) norm of the initial wave packet when it is expressed in product Jacobi coordinates, for several reactions. In all cases, the norm has an error smaller than $0.1 \%$.

\begin{tabular}{|c|c|c|c|c|c|c|c|c|c|c|c|c|c|}
\hline \multirow[b]{2}{*}{$A+B C \rightarrow A B+C$} & \multirow[b]{2}{*}{$a$} & \multirow[b]{2}{*}{$c$} & \multirow[b]{2}{*}{$\cos \beta$} & \multirow[b]{2}{*}{$A$} & \multicolumn{3}{|c|}{ Option (A) } & \multicolumn{3}{|c|}{ Option (B) } & \multicolumn{3}{|c|}{ Product Jacobi coordinates } \\
\hline & & & & & $n_{r}$ & $n_{R}$ & $n_{\gamma}$ & $n_{r}$ & $n_{R}$ & $n_{\gamma}$ & $n_{r}$ & $n_{R}$ & $n_{\gamma}$ \\
\hline $\mathrm{Li}+\mathrm{FH} \rightarrow \mathrm{LiF}+\mathrm{H}$ & 0.27 & 0.05 & 0.01 & 3.65 & 400 & 448 & 50 & $>1000$ & 448 & 50 & 400 & 448 & 80 \\
\hline $\mathrm{H}+\mathrm{FLi} \rightarrow \mathrm{HF}+\mathrm{Li}$ & 0.05 & 0.27 & 0.01 & 19.73 & 400 & 448 & 80 & $>1000$ & 448 & 90 & 400 & 448 & 60 \\
\hline $\mathrm{H}+\mathrm{DD} \rightarrow \mathrm{HD}+\mathrm{D}$ & 0.33 & 0.50 & 0.17 & 2.53 & 256 & 256 & 80 & 420 & 256 & 80 & 256 & 256 & 80 \\
\hline $\mathrm{F}+\mathrm{HH} \rightarrow \mathrm{FH}+\mathrm{H}$ & 0.95 & 0.50 & 0.48 & 0.55 & 256 & 320 & 150 & 256 & 256 & 150 & 256 & 256 & 280 \\
\hline $\mathrm{H}+\mathrm{HF} \rightarrow \mathrm{HH}+\mathrm{F}$ & 0.50 & 0.95 & 0.48 & 1.05 & 256 & 256 & 260 & 256 & 256 & 260 & 256 & 256 & 200 \\
\hline $\mathrm{F}+\mathrm{HO} \rightarrow \mathrm{FH}+\mathrm{O}$ & 0.95 & 0.94 & 0.90 & 0.11 & 256 & 1024 & 260 & 256 & 420 & 260 & 512 & 512 & 280 \\
\hline
\end{tabular}




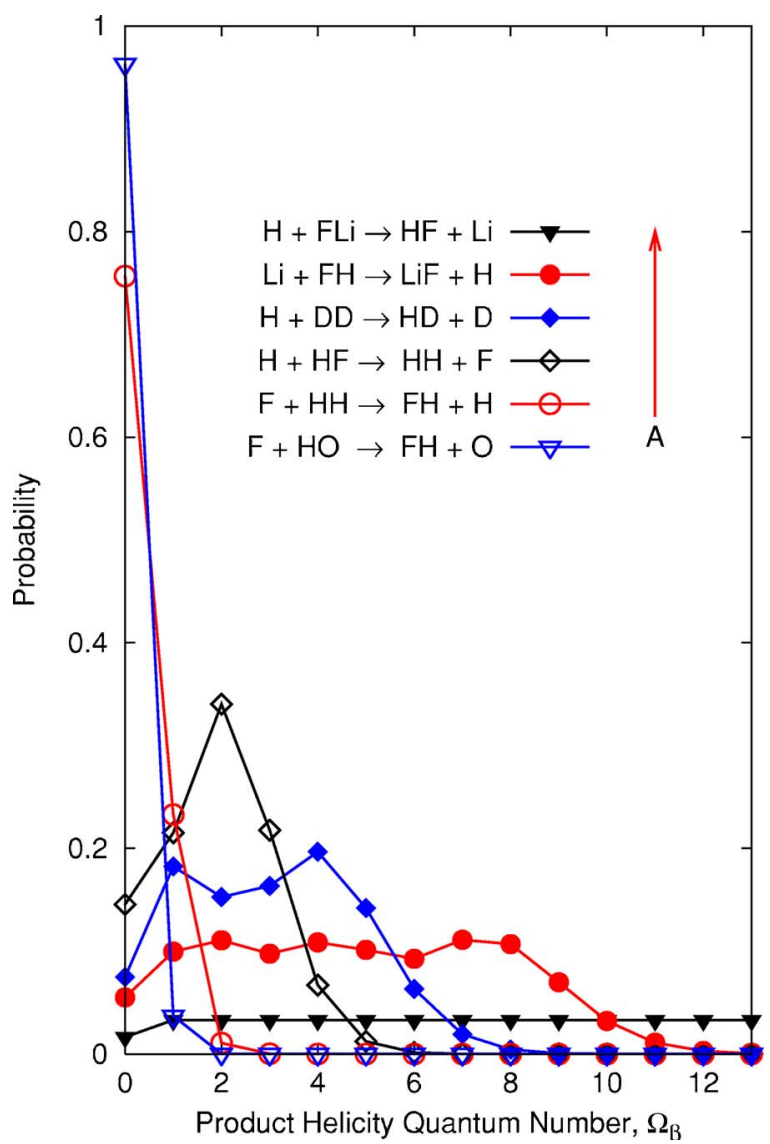

FIG. 4. Decomposition of an initial wave packet transformed to product Jacobi coordinates as a function of product helicity components $\Omega_{\beta}$ for $J$ $=30$. The $B C(v=0, j=0)$ initial state has been multiplied by an incoming complex gaussian at $R_{\alpha}=10 \AA$ for an incoming kinetic energy of $0.25 \mathrm{eV}$ and an energy spreading of $0.1 \mathrm{eV}$. Processes are in order of decreasing $A$ factor.

detail. For the $\mathrm{H}+\mathrm{D}_{2}$ example, $n_{R}$ [option (A)] $<n_{r}$ [option (B)], while for the $\mathrm{F}+\mathrm{H}_{2}$ is the reverse, as can be seen in Table I.

The situation changes for $\cos \beta$ factors close to unity, as in the case of $\mathrm{F}+\mathrm{OH}$ reaction, in which the narrower ring corresponds to the representation of option (A). In this limiting case the situation changes because $R_{\alpha} \approx R_{\beta}$. Thus, to properly describe the norm of the product wave functions, the minimum number of radial points required with option (A) is $\approx 1000$ while using option $(\mathrm{B}) \approx 400$ points are enough. The number of angular points remains, however, near the same being in this case rather large, $\approx 260$, because the product wave functions are very localized at $\gamma_{\alpha} \approx \pi$, as Fig. 2 shows.

In addition, the adequacy of the body-fixed frame should be analyzed to reduce the number of helicity components. Using reactant Jacobi coordinates, only one single $\Omega_{\alpha}$ projection is necessary to describe the initial wave packet. Thus, the total number of $\Omega_{\alpha}$ will depend on the strength of the Coriolis coupling term along the reaction dynamics. When using product Jacobi coordinates, however, and due to the body-fixed frame transformation, it is important to determine the number of helicity projections $\Omega_{\beta}$ required to describe the initial wave packet.

We consider the simplest case of $B C(v=0, j=0)$ reactant

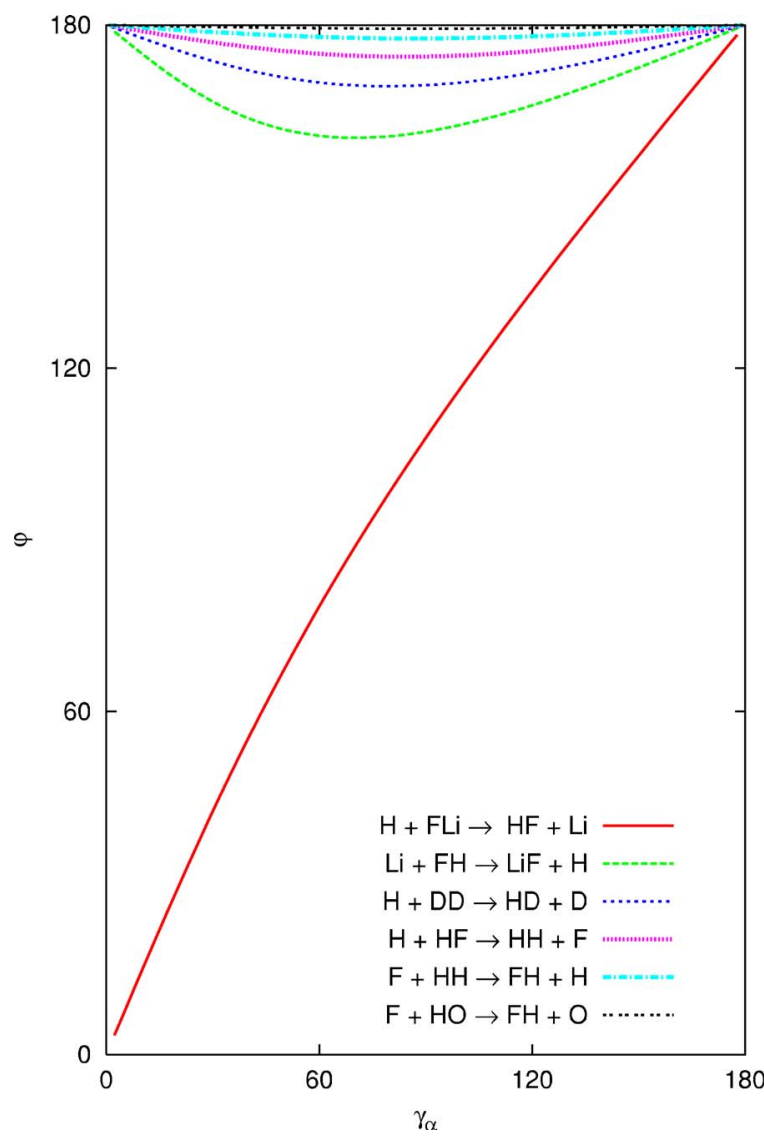

FIG. 5. Transformation angle $\varphi$ as a function of $\gamma_{\alpha}$, according to Eq. (24), for $r_{\alpha}$ fixed at the corresponding $B C$ equilibrium distance and $R_{\alpha}=10 \AA$.

state multiplied by an incoming Gaussian function located initially at $R_{\alpha}=10 \AA$ with a kinetic energies of 0.25 and $0.1 \mathrm{eV}$ of energy spreading. After the transformation to product Jacobi coordinates, the $\Omega_{\beta}$ distribution obtained is shown in Fig. 4. For large $A=a^{-1}-c$ factors (H+FLi case), the number of helicity components is very high. In fact, for this system all available $\Omega_{\beta}$ components are nearly equally probable. It is therefore more efficient to obtain state-to-state probabilities using reactant Jacobi coordinates and transforming to product coordinates using option (A).

As $A$ decreases, the number of $\Omega_{\beta}$ projections also decreases. In the limiting case of $A \approx 0$, the $\mathrm{F}+\mathrm{HO}$ case, the $z$ axis in reactant and product body-fixed frames is nearly parallel to the internuclear vector joining the two heavier atoms. For this reason $\Omega_{\beta}=0$ component is dominant in this case, and the efficiency of using reactant coordinates depends on the number of helicity projections required to converge the calculation in either reactant or product Jacobi coordinates.

These results are easily understood in terms of the angle $\varphi$ to rotate from the reactant to the product Jacobi frame given in Eq. (24). Restricting $r_{\alpha}$ to the $B C$ equilibrium distance and $R_{\alpha}$ to the mean distance of the incoming Gaussian, the angle $\varphi$ as a function of $\gamma_{\alpha}$ is shown in Fig. 5. For large $A, \varphi$ is proportional to $\gamma_{\alpha}$, varying from 0 to $\pi$. For low $A$ values, however, the available $\varphi$ are restricted to a narrow angular region around $\pi$, because $\mathbf{R}_{\alpha}$ and $-\mathbf{R}_{\beta}$ become nearly parallel. For $j=0, \Omega_{\alpha}=0$ the body-fixed frame transformation yields to nearly a single $\Omega_{\beta}=0$ projection because $d_{\Omega_{\beta}, 0}^{J} \propto \delta_{\Omega_{\beta}, 0}$ as $\varphi \rightarrow \pi$. 


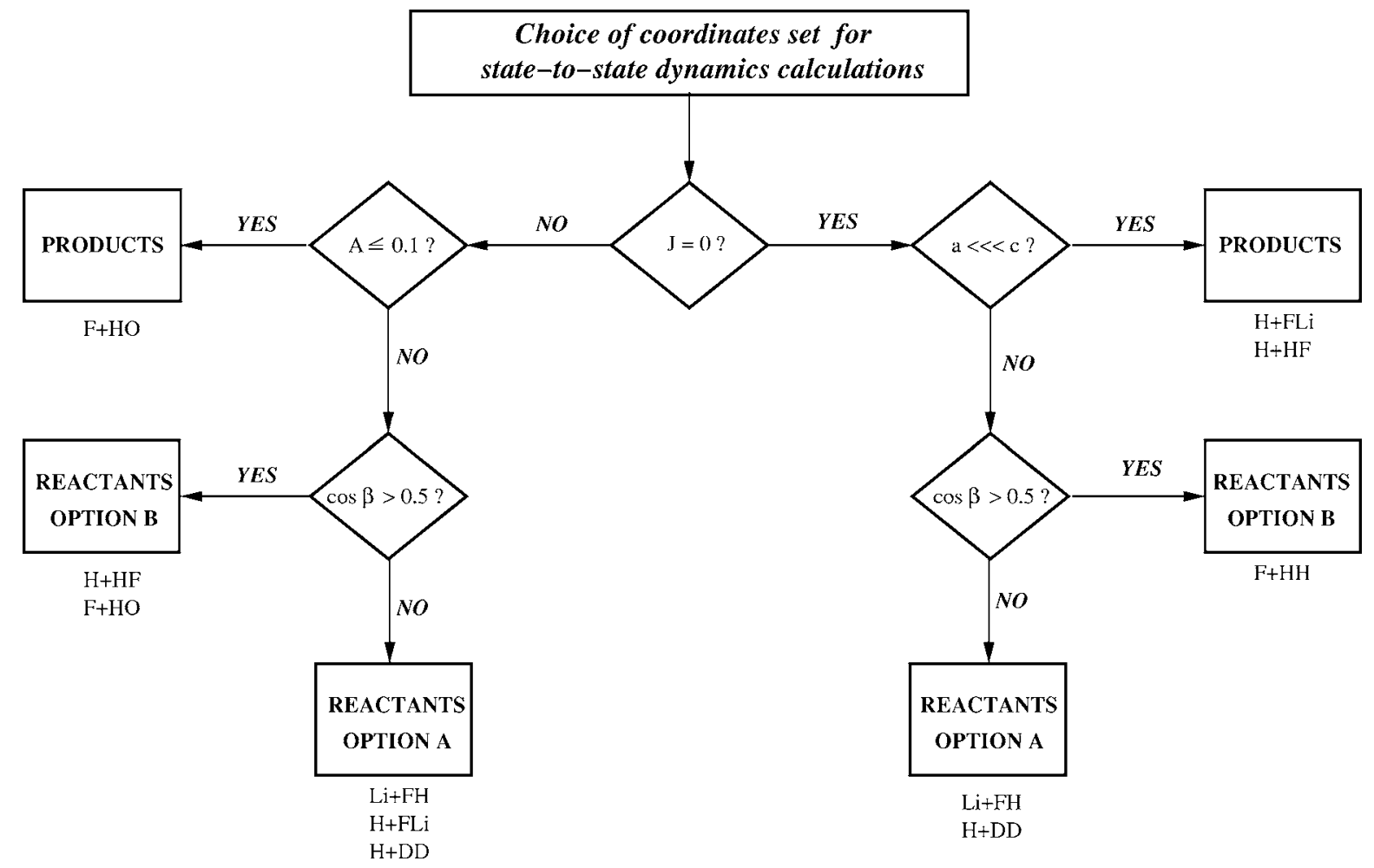

FIG. 6. Flowchart to choose the most suitable coordinate set to perform a state-to-state reaction dynamics calculation. The mass factors are $a=m_{A} /\left(m_{A}\right.$ $\left.+m_{B}\right), c=m_{C} /\left(m_{B}+m_{C}\right), \cos \beta=a, c$, and $A=a^{-1}-c$.

For $\mathrm{Li}+\mathrm{FH} \rightarrow \mathrm{LiF}+\mathrm{H}$, the use of reactant Jacobi coordinates presents the double advantage of yielding a significant reduction in the angular grid and the number of helicity components.

For the $\mathrm{H}+\mathrm{FLi} \rightarrow \mathrm{HF}+\mathrm{Li}$ reverse reaction, the number of helicity components is enormously reduced in reactant Jacobi coordinates, but since $c$ is low the use of "internal" product Jacobi coordinates is more efficient, as discussed above. For $J>0$, the use of reactant Jacobi coordinates and option (A) is expected to yield a significant advantage.

For intermediate systems, such as $\mathrm{H}+\mathrm{D}_{2}$ and $\mathrm{F}+\mathrm{H}_{2}$, using reactant coordinates is favorable, specially if the reduction introduced by considering the permutation of identical nuclei is considered, together with low helicity mixing in fast direct reactions.

In the case of low $A$, as it is the case for $\mathrm{F}+\mathrm{HO} \rightarrow \mathrm{FH}$ $+\mathrm{O}$, the use of reactant and product Jacobi coordinates is $a$ priori nearly equivalent according to the two arguments given, so that its choice depends on convergency details of the wave packet dynamics, as it is studied below. In Table I, all these arguments in favor or against the use of reactant/ product Jacobi coordinates are expressed in terms of the number of grid points and helicity components needed to reproduce the norm of the initial wave packet or product state wave functions.

In Fig. 6 flow chart shows the procedure to choose a given method based only on the masses of the atoms involved in the reaction, summarizing all the arguments discussed above.

The role of the reaction dynamics has to be taken into account in the convergency of the grids as is described below for the limiting cases of $\mathrm{Li}+\mathrm{FH}$ and $\mathrm{F}+\mathrm{HO}$ collisions. All these cases correspond to direct-like reactions with a barrier, even when many resonant structures appear. For such reaction mechanism, simple kinematic models can be used ${ }^{54-56}$ to understand product state distributions depending on the mass combination and the features of the transition state region around the saddle point where the reaction takes place. The energy partitioning, among translational, vibrational, and rotational, can then be approximately explained in terms of the mass factors used here. For insertion reaction mechanisms involving long lived complexes, however, the final energy partitioning of products is governed by statistical arguments as described elsewhere. ${ }^{57-60}$

\section{STATE-TO-STATE REACTION PROBABILITY RESULTS}

The total reaction probability is calculated using the flux method to check the accuracy of the method of calculation of the individual state-to-state probabilities. In addition, the inelastic $S$-matrix terms are also calculated to double check the total flux. In all cases the results have been converged to get an agreement better than $1 \%$.

The flux towards products can be obtained at any distance and is therefore easily converged. However, obtaining individual state-to-state probability requires to integrate far enough to reach the asymptotic region. If this is not the case, the sum of all state-to-state probabilities deviates from the total reaction probability obtained through the flux method. As $R_{\beta}^{\infty}$ increases, the number of grid points increases, specially when using reactant Jacobi coordinates because the density of grids points should also be increased to get good norms of product states. 
TABLE II. Parameters used in the dynamics calculations using reactant Jacobi coordinates (RJC) and product Jacobi coordinates (PJC). Distances are given in $\AA$. The damping function used $f(x)$ takes the forms $f(x)=1.0$ (if $x<x_{\text {abs }}$ ) and $f(x)=\exp \left[-\alpha_{\mathrm{abs}}\left(x-x_{\mathrm{abs}}\right)^{2}\right]$ (if $x>x_{\mathrm{abs}}$ ), with $x=r, R$.

\begin{tabular}{|c|c|c|c|}
\hline & \multirow{2}{*}{$\frac{\mathrm{Li}+\mathrm{FH}}{\mathrm{RJC}}$} & \multicolumn{2}{|c|}{$\mathrm{F}+\mathrm{HO}$} \\
\hline & & RJC & PJC \\
\hline$r_{\min } / r_{\max }$ & $0.25 / 17.5$ & $0.40 / 13.0$ & $0.40 / 13.0$ \\
\hline$R_{\min } / R_{\max }$ & $0.50 / 17.5$ & $0.40 / 14.0$ & $0.75 / 13.0$ \\
\hline$n_{r}$ & 400 & 512 & 256 \\
\hline$n_{R}$ & 512 & 420 & 420 \\
\hline$n_{\gamma}$ & 50 & 280 & 280 \\
\hline$R_{\beta}^{\infty}$ & 10.0 & 9.0 & 9.0 \\
\hline$r_{\mathrm{abs}} / \alpha_{\mathrm{abs}}$ & $13.0 / 0.017$ & $11.0 / 0.010$ & $11.0 / 0.010$ \\
\hline$R_{\mathrm{abs}} / \alpha_{\mathrm{abs}}$ & $14.5 / 0.025$ & $11.0 / 0.035$ & $11.0 / 0.035$ \\
\hline
\end{tabular}

Finally, individual state-to-state reaction probabilities are analyzed because their convergency requires larger grids and more helicity components than for the total reaction probability. After a convergency study, the grid parameters used in the calculations described below are listed in Table II. The number of iterations varies from case to case and typically is finished when more than $\approx 99 \%$ of the wave packet is absorbed.

\section{A. The $\mathrm{Li}+\mathrm{FH} \leftrightarrow \mathrm{LiF}+\mathrm{H}$ reaction}

This reaction can be considered to be thermoneutral: The potential energy surface is slightly endoergic while it becomes slightly exoergic when zero point energies are considered. The PES presents a late barrier at HF distances elongated with respect to the bare HF reactant, ${ }^{49}$ and at the saddle point, the reaction coordinate coincides with HF stretch. As a consequence, the reaction probability is highly enhanced by initial vibrational excitation of $\mathrm{HF}$ reactant, as found in collisions $^{61}$ and photoinitiated processes, ${ }^{62,63}$ in agreement with Polanyi's rules. ${ }^{64,65}$ The reaction presents a dynamical threshold at very low kinetic energies of $\approx 0.07 \mathrm{eV}$, associated to the zero point energy at the saddle point. The total reaction cross section ${ }^{61}$ and the vibrationally resolved reaction probabilities ${ }^{28}$ present oscillations as a function of translational energy which could be associated to the vibrational structure at the transition state region. Finally, the saddle point corresponds to a bent geometry, at an angle of $\approx \pi / 2$, in product Jacobi coordinates. Such feature produces that the final rotational distribution of $\mathrm{LiF}$ products presents a clear preference to produce even $j$ 's and nearly no odd $j$ 's for low rotational excitation. This direct link between transition state and final rotational distribution is based on the assumption of a fast direct mechanism once the transition state is reached, consistent with the direct interaction with product repulsion (DIPR) mechanism for these kind of harpoon-like reactions: ${ }^{66,67}$ One electron of the metal atom $(M)$ "jumps" towards the halide molecule $(\mathrm{HX})$, forming an unstable anion which fragments, leading to $M X$ products.

The state-to-state reaction probabilities for the $\mathrm{Li}$ $+\mathrm{FH}(v=0,1, j=0) \rightarrow \operatorname{LiF}\left(v^{\prime}, j^{\prime}\right)+\mathrm{H}$ collisions at $J=0$ were obtained and discussed previously using bond coordinates ${ }^{28}$ and the PES of Ref. 49. In this work, they have been recal-

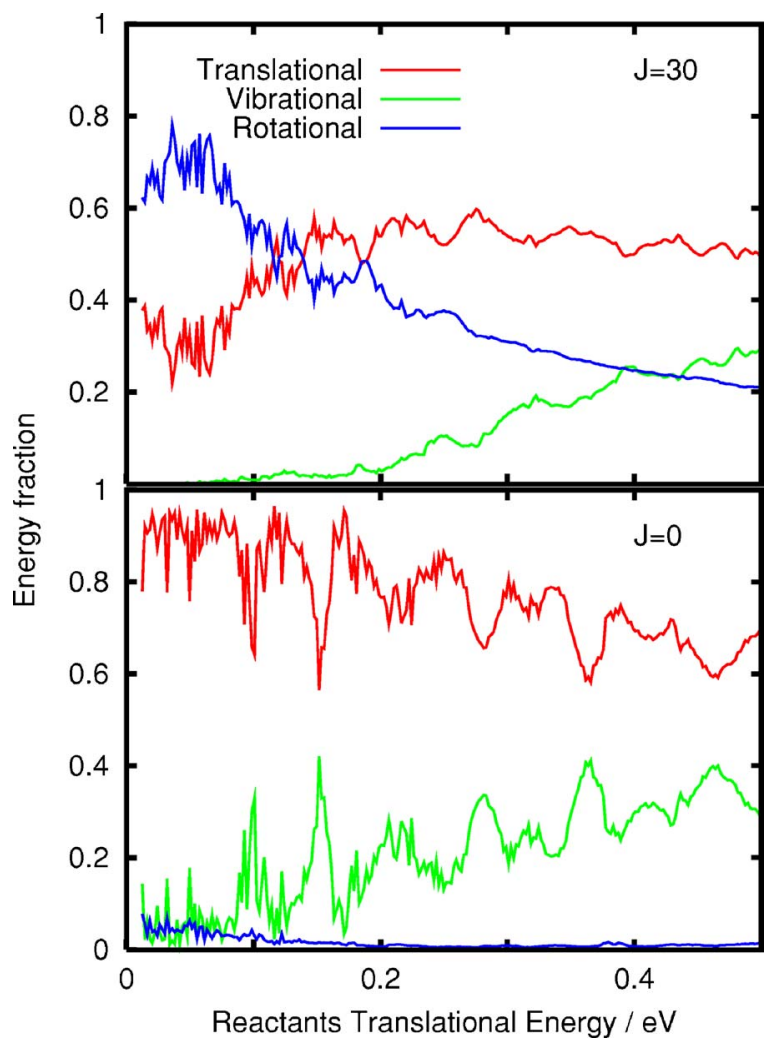

FIG. 7. Average values of the translational, vibrational, and rotational energy fractions of the $\operatorname{LiF}\left(v^{\prime}, j^{\prime}\right)$ products in the $\operatorname{Li}+\mathrm{HF}(v, j)$ collisions for $J=0$ (bottom panel) and $J=30$ (top panel).

culated using reactant Jacobi coordinates, giving results in perfect agreement (better than 1\%) with those previously reported. ${ }^{28}$ For $J=0$ it is found that the energy fraction goes mainly to translation (more than 3/4), 20\%-25\% goes to vibration, while the rotational energy fraction is lower than $10 \%$, as can be seen in Fig. 7. In this case $r_{\beta} \approx R_{\alpha}$ at the saddle point, which suggests that product vibrational energy is connected to reactant translational energy. In fact, for $J$ $=0$, the vibrational energy fraction increases with initial translational energy.

For $J>0$, the situation changes. In this case reactant orbital angular momentum $\ell$ essentially transforms to product rotational angular momentum $\mathbf{j}^{\prime}$, as it is demonstrated using kinematic models. ${ }^{56}$ Therefore, for $j=0$ considered here, total angular momentum $(\ell=\mathbf{J}-j)$ is expected to transform in product rotational angular momentum, thus producing a considerable rotational excitation as compared with $J$ $=0$, as shown in Fig. 7. The rotational energy fraction for $J$ $=30$ is very similar to the values obtained in quassiclassical simulations, $\approx 36 \%,{ }^{68}$ and with the angular constraint model by Bonnet and Rayez applied to this system, $\approx 38 \%$.

The vibrationally resolved reaction probabilities for the $\mathrm{Li}+\mathrm{FH}(v=0, j=0) \rightarrow \mathrm{LiF}\left(v^{\prime}\right)+\mathrm{H}$ collisions for $J=0$, in the bottom panel of Fig. 8, present broad oscillations as a function of translational energies. The maxima of the oscillations are approximately equidistant and were interpreted as due to quasibound states at the saddle point. ${ }^{61}$ At low energies there are also many resonances attributed to a relatively deep well in the entrance channel. These resonances tend to disappear for $J=30$, in the top panel of Fig. 8, by two reasons: first, the 


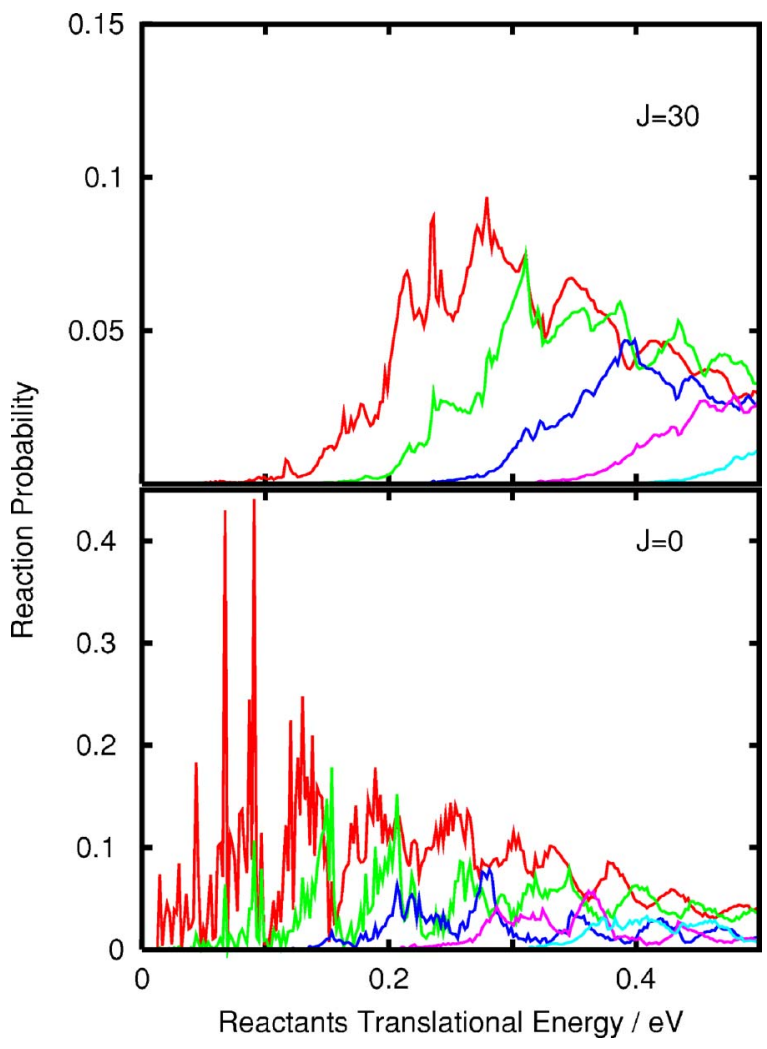

FIG. 8. Vibrationally resolved state-to-state reaction probabilities for $\mathrm{Li}$ $+\mathrm{HF}(v=0, j=0) \rightarrow \operatorname{LiF}\left(v^{\prime}\right)+\mathrm{H}$ collisions at $J=0$ (bottom panel) and 30 (top panel), as a function of reactants translational energy.

rotational barrier "closes" the reaction channel at low energies and shifts resonances towards higher energies where they become broader, and, second, the near degenerate rotational sublevels interact among them and new dissociation channels appear, which gives rise to many more resonances much broader than for $J=0$. The broad oscillations also tend to disappear, because different $\Omega_{\alpha}$ channels present different oscillatory patterns.

The increase of total angular momentum has a much dramatic effect on the rotational distributions. In the bottom panel of Fig. 9 the probabilities of forming $\operatorname{LiF}\left(v^{\prime}=0, j^{\prime}\right)$ are shown for collisions at a translational energy of $\approx 0.2 \mathrm{eV}$ and for $J=0$ and 30 . For $J=0$ the $\operatorname{LiF}\left(j^{\prime}\right)$ rotational distribution is peaked at low even $j^{\prime}$, essentially $j^{\prime}=0$ and 2. For $J=30$ this distribution is very much shifted towards higher $j^{\prime}$ values, $j^{\prime} \approx 30$. This fact implies the use of larger grids as compared with those used for $J=0$.

The reaction probabilities for the $\mathrm{Li}+\mathrm{FH}(v=0, j=0, J$ $=30) \rightarrow \operatorname{LiF}\left(v^{\prime}=0\right)+\mathrm{H}$ collisions as a function of the helicity quantum number $\Omega_{\beta}$, in the top panel of Fig. 9 , present a very broad distribution. This would imply the inclusion of all $\Omega_{\beta}$ components in dynamical calculations if product Jacobi coordinates were used. However, using reactant jacobi coordinates the convergence is reached by including much less $\Omega_{\alpha}$ projections. As an example, in Fig. 9 the rotationally resolved reaction probabilities are shown for calculations with an increasing number of $\Omega_{\alpha}$ components. Therefore, the reduction in the number of helicity components, by a factor of $\approx 3$, clearly demonstrates the larger advantage of using reactant coordinates.
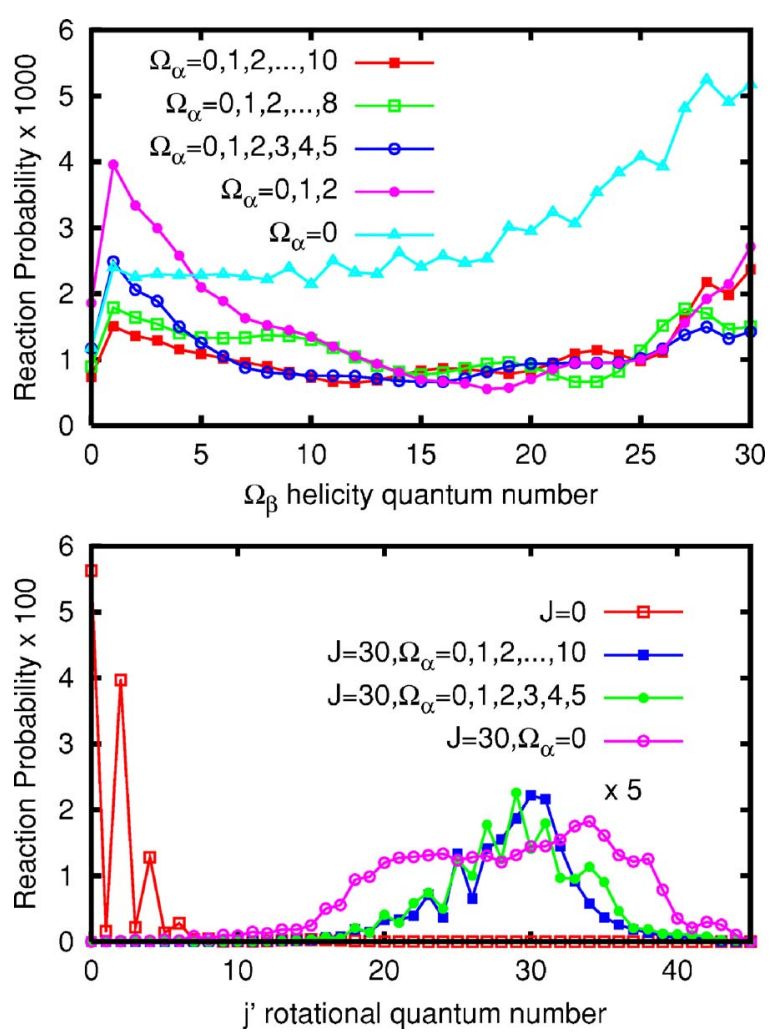

FIG. 9. Reaction probabilities of $\operatorname{LiF}\left(v^{\prime}=0, j^{\prime}\right)$ (bottom panel) and $\operatorname{LiF}\left(v^{\prime}\right.$ $\left.=0, \Omega_{\beta}\right)$ (top panel) obtained in the $\operatorname{Li}+\mathrm{FH}(v=0, j=0)$ collision at a translational energy of $0.2 \mathrm{eV}$ and $J=0$ and 30. For $J=30$, distributions are shown for several calculations including an increasing number of $\Omega_{\alpha}$ components.

\section{B. The $\mathrm{F}+\mathrm{HO} \rightarrow \mathrm{FH}+\mathrm{O}$ reaction}

The $\mathrm{F}\left({ }^{2} P\right)+\mathrm{HO}\left({ }^{2} \Pi\right) \rightarrow \mathrm{FH}\left({ }^{1} \Sigma^{+}\right)+\mathrm{O}\left({ }^{3} P\right)$ reaction is very exoergic, by $\approx 2 \mathrm{eV}$, with the $\mathrm{HF}\left(v^{\prime}=3\right)$ products being nearly isoenergetic with $\mathrm{OH}(v=0)$ reactants. The PES of the $1{ }^{3} A^{\prime \prime}$ ground electronic state has been recently proposed. ${ }^{69}$ It presents a low barrier at a bent geometry and two wells: one in the entrance channel with a bent geometry and a second one in the products channel with a linear geometry. The reaction presents two different mechanisms: ${ }^{69}$ one direct at energies above the transition state zero point energy, at $\approx 0.1 \mathrm{eV}$, and below this threshold a second indirect mediated by $H L H$ resonances. ${ }^{69,70}$ These resonances are associated to periodic orbits at the transition state and also appeared in quasiclassical simulations. ${ }^{69}$ The resonances survive for rather high total angular momentum so that the total reaction cross section presents also two parts, associated to these two mechanisms.

In this case, the light atom is exchanged among the two heavier ones. It can be considered that $R_{\beta} \approx R_{\alpha}$, suggesting that the initial translational energy between reactant is converted in translational energy between products. However, because of the large exoergicity, some important changes must be expected. In fact, the energy seems to be nearly equally distributed among translation and vibration of products, with very little excitation of rotations, see Fig. 10. The larger rotational constant corresponds to the diatomic molecule, formed by a light $\mathrm{H}$ atom and a much heavier atom such as $\mathrm{O}$ or $\mathrm{F}$, and most of the rotational energy is carried 


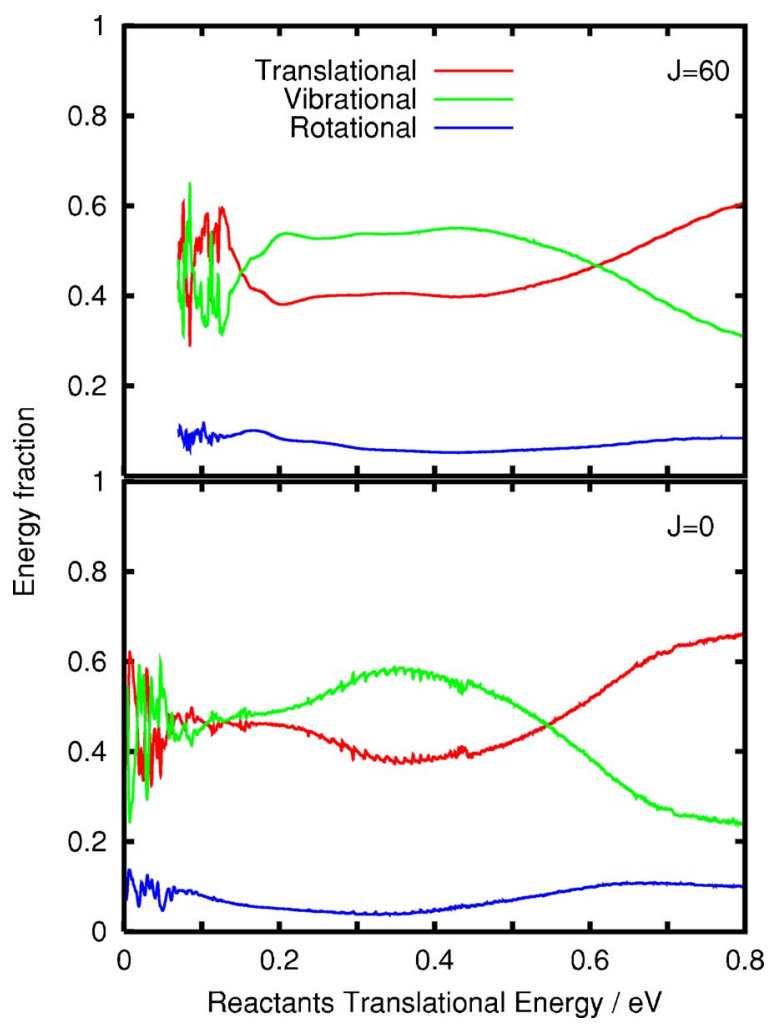

FIG. 10. Average values of the translational, vibrational, and rotational energy fractions of the $\mathrm{HF}\left(v^{\prime}, j^{\prime}\right)$ products in the $\mathrm{F}+\mathrm{HO}(v=0, j=0)$ collisions for $J=0$ (bottom panel) and $J=60$ (top panel).

by $\mathrm{OH}$ or HF. In this case, $\ell \approx \ell^{\prime}$ and their rotational constants are relatively very low. Assuming that the heavy atoms rotate slowly, most of the rotational motion can be attributed to the $\mathrm{H}$ atom. As a consequence, the rotational angular momentum of $\mathrm{OH}$ transforms into angular momentum of $\mathrm{HF}$ products, which presents a very similar rotational constant of $\approx 2 \mathrm{meV}$. This situation seems to be independent of the total angular momentum, as can be seen in the top panel of Fig. 10 , in contrast to the $\mathrm{Li}+\mathrm{FH}$ case.

In order to analyze in more detail the dynamics, in Fig. 11 the vibrationally resolved reaction probabilities are shown for $J=0$ and $J=60$. At $J=0$ there are many resonances below the threshold at $0.1 \mathrm{eV}$ (shown in detail in the inset of the figure), which nearly disappear at $J=60$. The threshold of $\approx 0.1 \mathrm{eV}$ is associated to the zero point energy in the transition state. ${ }^{69}$ It was found that, at the saddle point, the reaction coordinate essentially corresponds to the HF stretch. If products are reached following a direct mechanism once the system has reached the saddle point, the increase of $v^{\prime}$ would imply an increase in the HF energy at the transition state. Assuming that the perpendicular modes are approximately in their ground state, such simplified image would explain why the main maxima in the $v^{\prime}$ distribution (at 0.1, 0.2, and $0.4 \mathrm{eV}$ for $v^{\prime}=1,2$, and 3, respectively) appear at increasing total energy. Finally, the secondary maxima (at $E \approx 0.1 \mathrm{eV}$ for $v^{\prime}=3$, for example) would be due to a redistribution of energy in the exit channel.

The final rotational probabilities of HF product, shown in bottom panel of Fig. 12 for $v^{\prime}=1$, change only slightly when increasing $J$. For $J=60$, it becomes broader and some
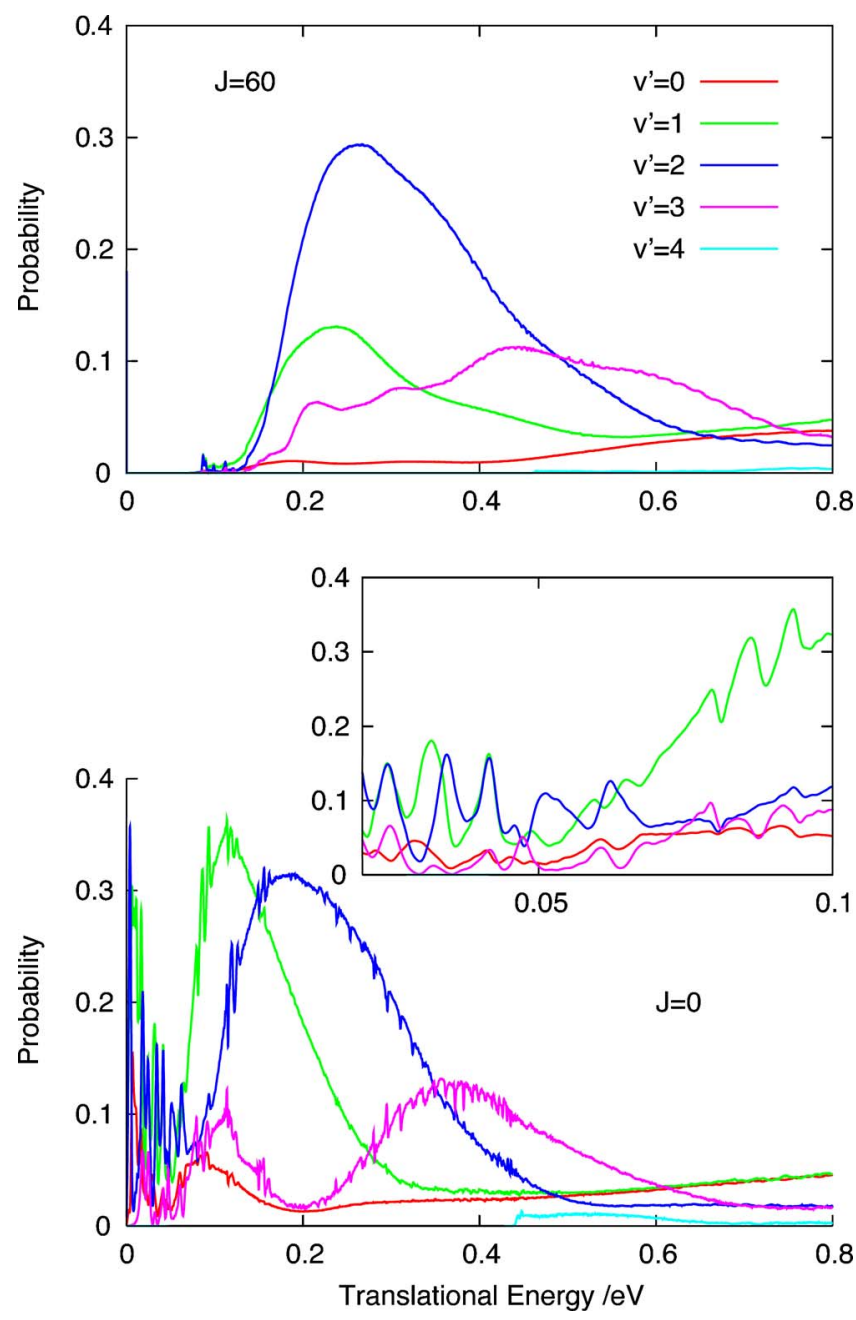

FIG. 11. Vibrationally resolved state-to-state reaction probabilities for $\mathrm{F}$ $+\mathrm{HO}(v=0, j=0) \rightarrow \mathrm{FH}\left(v^{\prime}\right)+\mathrm{O}$ collision at $J=0$ (bottom panel) and $J=60$ (top panel).A detail at low energies for $J=0$ is shown in the middle panel.

structures are lost. The dependence of the final rotational probability with energy is similar for both $J$ 's, apart from the natural energy shift introduced by the rotational barrier and the disappearance of the resonances at low energies. The final $\operatorname{HF}\left(v^{\prime}=1, j^{\prime}=0\right)$ probabilities as a function of energy for an increasing number of $\Omega_{\beta}$ components (in product Jacobi coordinates) are presented in the top panel of Fig. 12 to show the evolution of the convergency.

It is important to note the overall convergency of the reaction probability as a function of the number of helicity components either in reactant or product Jacobi coordinates, as it is shown in Fig. 13 for $J=60$. In the two cases, the number of helicity components required to converge the calculations is pretty low, showing the good adequacy of centrifugal sudden approximations. This is expected since the two body-fixed $z$ axes nearly coincide with the heavy atoms internuclear vector. For low energies below $0.2 \mathrm{eV}$, the presence of resonances facilitates the $\Omega$ mixing that makes that the number of helicity components needed pretty similar in the two body-fixed frames, $\approx 2$ for reactants and $\approx 3$ for products. At higher energies, however, the direct mechanism yields to an important decoupling of the helicity components. Thus, in the reactant frame the total reaction probabilities are 


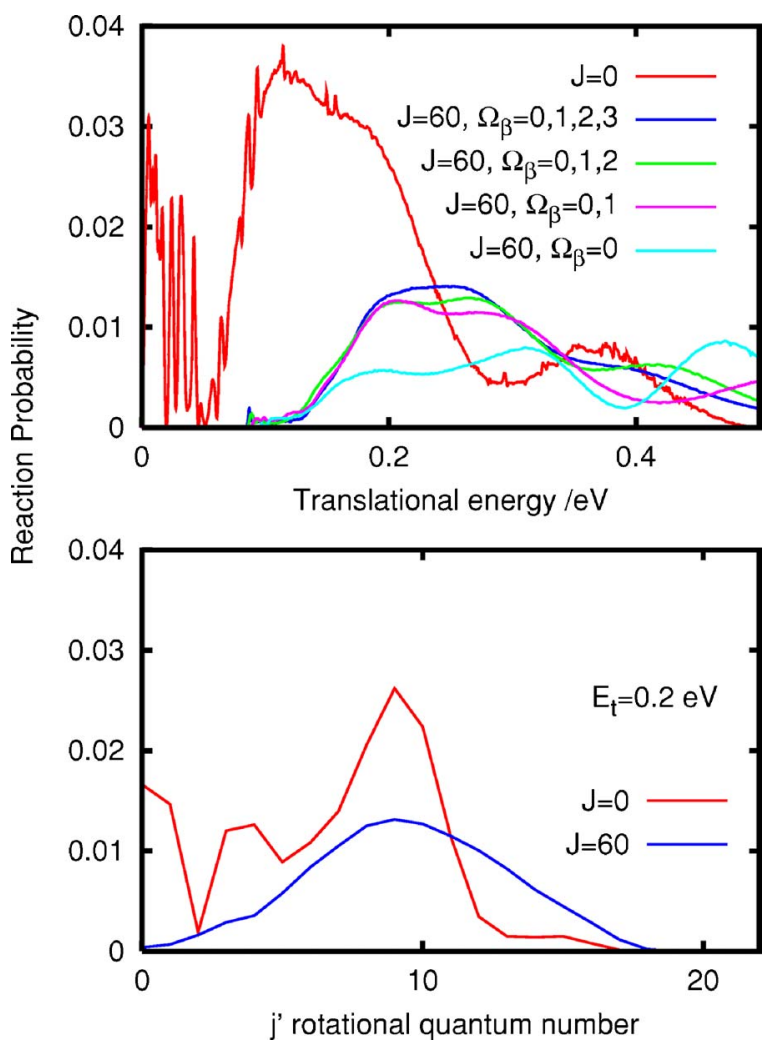

FIG. 12. Rotationally resolved state-to-state reaction probabilities for $\mathrm{F}$ $+\mathrm{HO}(v=0, j=0) \rightarrow \mathrm{FH}\left(v^{\prime}=1, j^{\prime}\right)+\mathrm{O}$ collision at $J=0$ and 60 . Bottom panel, at a translational energy of $0.2 \mathrm{eV}$; top panel, for $j^{\prime}=9$ as a function of translational energy. For $J=60$, results for an increasing number of $\Omega_{\beta}$ (using product Jacobi coordinates) are shown to analyze the convergency.

very similar in all cases, and the calculation can be considered to be rather well converged by simply including $\Omega_{\alpha}$ $=0$ and 1. However, in the product frame this is not so, probably because the initial wave packet needs at least up to $\Omega_{\beta}=3$ to reach convergency. This reduction by a factor of 2 in the helicity components compensates for the fact that using reactant coordinates more dense grids are needed to converge the calculations (see Table II).

In a recent work, the state-to-state cross section has been calculated for the $\mathrm{O}\left({ }^{3} P\right)+\mathrm{HCl} \rightarrow \mathrm{OH}+\mathrm{Cl}$ (Ref. 71) using a time-independent approach with democratic hyperspherical coordinates. $^{72}$ This system is heavier than the $\mathrm{OH}+\mathrm{F}$ studied here, but the exoergidicity is much lower. This last fact requires the inclusion of many more channels in the $\mathrm{OH}+\mathrm{F}$ case, making the calculations more difficult.

For this reason the use of elliptic hyperspherical coordinates seems more adequate for $\mathrm{OH}+\mathrm{F}$. $^{73-77}$ And recently the state-to-state probabilities for $J=0$ have been calculated using these coordinates, ${ }^{78}$ showing a good agreement with the present results. The low number of components required to converge the calculation using reactant Jacobi coordinates justifies the use of the reactant Jacobi body-fixed frame as it is done in the elliptic hyperspherical approach. ${ }^{73,76,77}$

Using product Jacobi coordinates, some more helicity components are needed to converge the reaction probability. This is probably due to the necessity of including $\Omega_{\beta}=0,1$, and 2 to converge the norm of the initial wave packet with an error lower than $1 \%$. However, the grid for reactant coordi-

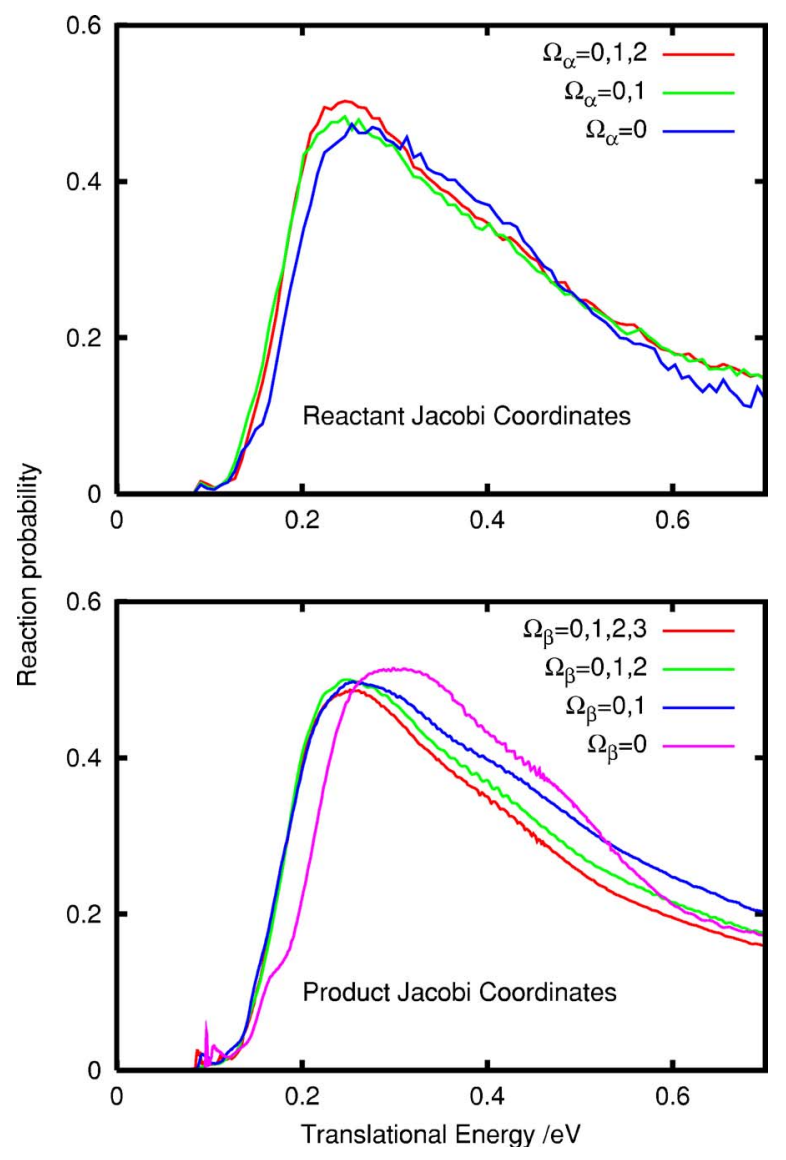

FIG. 13. Total reaction probabilities for $\mathrm{F}+\mathrm{HO}(v=0, j=0) \rightarrow \mathrm{FH}+\mathrm{O}$ at $J$ $=60$ as a function of the translational energy obtained either in product (bottom panel) and reactant (top panel) Jacobi coordinates. In each case, the different curves correspond to calculation including an increasing number of helicity components.

nates needs more points than for product coordinates. In conclusion, for this system the use of product Jacobi coordinate becomes slightly more efficient for low energies, in contrast with all previous systems for which reactant Jacobi coordinates are clearly more efficient, specially for $J>0$. For high energies, however, the situation is reverse, with reactant Jacobi coordinates being slightly more efficient.

\section{CONCLUSIONS}

In this work a procedure has been proposed for the transformation from reactant to product Jacobi coordinates designed for the extraction of state-to-state reaction probabilities using a time-dependent method in a body-fixed frame. The method developed consists of a series of steps and requires a computation that is time negligible with that associated to each iteration of the time propagation. Thus, its efficiency with respect to the most commonly used product Jacobi coordinates depends on the size of the grids and basis set functions used.

This is studied for several systems to analyze the effect of the mass combinations involved in the reaction, through the use of the mass factors $a$ and $c$, separated in two aspects: the description of the initial wave packet and convergency of time-dependent calculations. For zero total angular momentum, it is found that for $a \gg c$, reactant coordinates are more 
suited while for $a \ll c$ it is better to use product coordinates. When using reactant coordinates, the description of intermediate coordinates is done with option (A), for $\cos \beta<0.5$ or option (B) for $\cos \beta>0.5$. for $J>0$ it is found that the number of helicity components required to converge the norm of the initial wave packet is proportional to the mass factor $A$. This indicates that in general the use of reactant Jacobi coordinates is more efficient, unless $A \approx 0$. The election of the most suited set of coordinates and transformation method based on these mass factors is summarized in the flow chart of Fig. 6.

This is checked by the calculation of state-to-state reaction probabilities for several systems and several total angular momenta. All the reactions studied are direct, even when in some cases they present many resonant structures. The energy partition is also qualitatively explained in terms of the same factors.

In most of the cases, the use of reactant Jacobi coordinates requires a lower number of helicity components, thus showing their good adequacy for performing dynamical calculations. The only case in which both sets of coordinates are computationally very similar is for the $\mathrm{F}+\mathrm{HO}$ reaction.

\section{ACKNOWLEDGMENTS}

The authors want to thank Dr. B. Lepetit and Dr. A. Dupays for sending the authors the results prior to publication and very fruitful discussions. The authors also want to acknowledge very interesting discussions with Professor Pablo Villarreal and Dr. Tomas González-Lezana. The authors also want to deeply thank Adela Fernandez de Lama for her help and enthusiasm. This work has been supported by DGICYT (Ministerio de Educación y Ciencia, Spain) under Grant No. CTQ2004-02415/BQU The traveling CSICCNRS Grant Nos. 2004FR0003 and 2005FR0031 are also acknowledged.

\section{APPENDIX: VOLUME ELEMENTS}

The volume element in the Jacobi coordinates considered is given by

$$
\begin{aligned}
d V & =\sqrt{\left|g_{\xi}\right|} d R_{\xi} d r_{\xi} d \gamma_{\xi} d \phi_{\xi} d \theta_{\xi} d \chi_{\xi} \quad \text { with } \sqrt{\left|g_{\xi}\right|} \\
& =R_{\xi}^{2} r_{\xi}^{2} \sin \gamma_{\xi} \sin \theta_{\chi},
\end{aligned}
$$

where $g_{\xi}$ is the $6 \times 6$ metric matrix, ${ }^{79}$ while $\left|g_{\xi}\right|$ is its determinant. In the new intermediate coordinates of option (A), $\left(R_{\beta}, R_{\alpha}, \gamma_{\alpha}, \phi_{\alpha}, \theta_{\alpha}, \chi_{\alpha}\right)$ as an example, the new metric matrix is given by

$$
g_{A}^{\prime}=\mathcal{J}_{A} \cdot g_{\alpha} \cdot \mathcal{J}_{A}^{T},
$$

where $\mathcal{J}_{A}$ is the Jacobian matrix of the transformation. In this new coordinate system, the volume element is analogously given by

$$
d V^{\prime}=\sqrt{\left|g_{A}^{\prime}\right|} d R_{\beta} d R_{\alpha} d \gamma_{\alpha} d \phi_{\alpha} d \theta_{\alpha} d \chi_{\alpha}
$$

where $\sqrt{\left|g^{\prime}{ }_{A}\right|}=\sqrt{\left|g_{\alpha}\right|}\left|\mathcal{J}_{A}\right|$ and the determinant of the Jacobian is given by

$$
\left|\mathcal{J}_{A}\right|=\frac{\partial r_{\alpha}}{\partial R_{\beta}}=\frac{\left(\left(m_{0}+m_{2}\right) / m_{2}\right)^{2} R_{\beta}}{A \sqrt{R_{\alpha}^{2}\left(\cos ^{2} \gamma_{\alpha}-1\right)+\left[\left(m_{0}+m_{2}\right) R_{\beta} / m_{2}\right]^{2}}} .
$$

In a similar way, for the second transformation option (B), $\left(R_{\beta}, r_{\alpha}, \gamma_{\alpha}, \phi_{\alpha}, \theta_{\alpha}, \chi_{\alpha}\right) \sqrt{\left|g^{\prime}{ }_{B}\right|}=\sqrt{\left|g_{\alpha}\right|}\left|\mathcal{J}_{B}\right|$, with

$$
\left|\mathcal{J}_{B}\right|=\frac{\partial R_{\alpha}}{\partial R_{\beta}}=\frac{\left(\left(m_{0}+m_{2}\right) / m_{2}\right)^{2} R_{\beta}}{\sqrt{\left(A r_{\alpha}\right)^{2}\left(\cos ^{2} \gamma_{\alpha}-1\right)+\left[\left(m_{0}+m_{2}\right) R_{\beta} / m_{2}\right]^{2}}} .
$$

${ }^{1}$ P. Casavecchia, N. Balucani, and G. G. Volpi, Annu. Rev. Phys. Chem. 50, 347 (1999).

${ }^{2}$ K. Liu, Annu. Rev. Phys. Chem. 52, 139 (2001).

${ }^{3}$ X. Yang, Int. Rev. Phys. Chem. 24, 37 (2005).

${ }^{4}$ S. C. Althorpe and D. C. Clary, Annu. Rev. Phys. Chem. 54, 493 (2003).

${ }^{5}$ A. Kuppermann and P. G. Hipes, J. Chem. Phys. 84, 5962 (1986).

${ }^{6}$ R. T. Pack and G. A. Parker, J. Chem. Phys. 87, 3888 (1987).

${ }^{7}$ J. M. Launay and M. Le Dourneuf, Chem. Phys. Lett. 163, 178 (1989).

${ }^{8}$ B. Lepetit and J. M. Launay, J. Chem. Phys. 95, 5159 (1991).

${ }^{9}$ J. F. Castillo, D. E. Manolopoulos, K. Stark, and H.-J. Werner, J. Chem. Phys. 104, 6531 (1996).

${ }^{10}$ M. P. de Miranda, D. C. Clary, J. F. Castillo, and D. E. Manolopoulos, J. Chem. Phys. 108, 3142 (1998).

${ }^{11}$ S. K. Pogrebnya, J. Palma, D. C. Clary, and J. Echave, Phys. Chem. Chem. Phys. 2, 693 (2000).

${ }^{12}$ J. Z. H. Zhang, and W. H. Miller, J. Chem. Phys. 91, 1528 (1989).

${ }^{13}$ D. G. Truhlar, D. W. Schwenke, and D. J. Kouri, J. Phys. Chem. 94, 7346 (1990).

${ }^{14}$ N. Balakrishnan, C. Kalyanaraman, and N. Sathyamurthy, Phys. Rep. 280, 79 (1997).

${ }^{15}$ G. G. Balint-Kurti, Adv. Chem. Phys. 128, 249 (2004).

${ }^{16}$ W. Zhu, J. Dai, J. Z. H. Zhang, and D. H. Zhang, J. Chem. Phys. 105, 4881 (1996).

${ }^{17}$ D. H. Zhang, D. Q. Xie, M. H. Yang, and S. Y. Lee, Phys. Rev. Lett. 89, 283203 (2002).

${ }^{18}$ Y. Zhang, T. X. Xie, K. L. Han, and J. Z. H. Zhang, J. Chem. Phys. 124, 134301 (2006).

${ }^{19}$ K. Yuan, Y. Chen, X. Liu, S. Harich, X. Yang, and D. H. Zhang, Phys. Rev. Lett. 96, 103202 (2006).

${ }^{20}$ D. H. Zhang and J. C. Light, J. Chem. Phys. 105, 1291 (1996).

${ }^{21}$ S. C. Althorpe, J. Chem. Phys. 114, 1601 (2001).

${ }^{22}$ F. Gögtas, G. G. Balint-Kurti, and A. R. Offer, J. Chem. Phys. 104, 7927 (1996).

${ }^{23}$ T. Peng and J. Z. H. Zhang, J. Chem. Phys. 105, 6072 (1996).

${ }^{24}$ W. Zhu, D. Wang, and J. Z. H. Zhang, Theor. Chem. Acc. 96, 31 (1997).

${ }^{25}$ J. Dai and J. Z. H. Zhang, J. Chem. Soc., Faraday Trans. 93, 699 (1997).

${ }^{26}$ S. C. Althorpe, D. J. Kouri, and D. K. Hoffman, J. Chem. Phys. 107, 7816 (1997)

${ }^{27}$ D. Neuhauser and M. Baer, J. Chem. Phys. 91, 4651 (1989).

${ }^{28}$ M. Lara, A. Aguado, M. Paniagua, and O. Roncero, J. Chem. Phys. 113, 1781 (2000).

${ }^{29}$ D. Neuhauser, M. Baer, R. S. Judson, and D. J. Kouri, J. Chem. Phys. 93, 312 (1990).

${ }^{30}$ R. S. Judson, D. J. Kouri, D. Neuhauser, and M. Baer, Phys. Rev. A 42, 351 (1990).

${ }^{31}$ R. N. Zare, Angular Momentum (Wiley, New York, 1988).

${ }^{32}$ O. Roncero, D. Caloto, K. C. Janda, and N. Halberstadt, J. Chem. Phys. 107, 1406 (1997).

${ }^{33}$ Y. Huang, D. J. Kouri, and D. K. Hoffman, J. Chem. Phys. 101, 10493 (1994).

${ }^{34}$ V. A. Mandelshtam and H. S. Taylor, J. Chem. Phys. 103, 2903 (1995).

${ }^{35}$ Y. Huang, S. S. Iyengar, D. J. Kouri, and D. K. Hoffman, J. Chem. Phys. 105, 927 (1996).

${ }^{36}$ G.-J. Kroes and D. Neuhauser, J. Chem. Phys. 105, 8690 (1996).

${ }^{37}$ R. Chen and H. Guo, J. Chem. Phys. 105, 3569 (1996).

${ }^{38}$ S. K. Gray and G. G. Balint-Kurti, J. Chem. Phys. 108, 950 (1998).

${ }^{39}$ H. Tal-Ezer and R. Kosloff, J. Chem. Phys. 81, 3967 (1984).

${ }^{40}$ J. C. Light, I. P. Hamilton, and J. V. Lill, J. Chem. Phys. 82, 1400 (1985).

${ }^{41}$ J. T. Muckerman, Chem. Phys. Lett. 173, 200 (1990). 
${ }^{42}$ D. T. Colbert and W. H. Miller, J. Chem. Phys. 96, 1982 (1992).

${ }^{43}$ R. Kosloff, J. Phys. Chem. 92, 2087 (1988).

${ }^{44}$ G. G. Balint-Kurti, R. N. Dixon, and C. C. Marston, J. Chem. Soc., Faraday Trans. 86, 1741 (1990).

${ }^{45}$ G. C. Schatz and A. Kuppermann, J. Chem. Phys. 65, 4642 (1976).

${ }^{46}$ Y. Sun, R. S. Judson, and D. J. Kouri, J. Chem. Phys. 65, 226 (1976).

${ }^{47}$ Y. Sun, R. S. Judson, and D. J. Kouri, J. Chem. Phys. 90, 241 (1989).

${ }^{48}$ R. D. Levine and R. B. Bernstein, Molecular Reaction Dynamics and Chemical Reactivity (Oxford University Press, New York, 1987).

${ }^{49}$ A. Aguado, M. Paniagua, M. Lara, and O. Roncero, J. Chem. Phys. 107, 10085 (1997)

${ }^{50}$ A. I. Boothroyd, W. J. Keogh, P. G. Martin, and M. R. Peterson, J. Chem. Phys. 95, 4343 (1991).

${ }^{51}$ A. I. Boothroyd, W. J. Keogh, P. G. Martin, and M. R. Peterson, J. Chem. Phys. 104, 7139 (1996)

${ }^{52}$ K. Stark and H-J. Werner, J. Chem. Phys. 104, 6515 (1996).

${ }^{53}$ S. Gómez-Carrasco, L. González-Sánchez, A. Aguado, M. Paniagua, O. Roncero, M. L. Hernández, and J. M. Alvariño, Chem. Phys. Lett. 383, 25 (2004).

${ }^{54}$ L. Bonnet and J. C. Rayez, J. Chem. Phys. 102, 9512 (1995).

${ }^{55}$ L. Bonnet and J. C. Rayez, Phys. Chem. Chem. Phys. 1, 2383 (1999).

${ }^{56}$ K. Truhins, R. Marsh, A. J. McCaffery, and T. W. J. Whiteley, J. Chem. Phys. 112, 5281 (2000).

${ }^{57}$ W. Hauser and H. Feshbach, Phys. Rev. 87, 366 (1952).

${ }^{58}$ P. Pechukas and J. C. Light, J. Chem. Phys. 42, 3281 (1965).

${ }^{59}$ W. H. Miller, J. Chem. Phys. 52, 543 (1970).

${ }^{60}$ E. J. Rackhan, T. González-Lezana, and D. E. Manolopoulos, J. Chem. Phys. 119, 12895 (2003).

${ }^{61}$ M. Lara, A. Aguado, O. Roncero, and M. Paniagua, J. Chem. Phys. 109, 9391 (1998)

${ }^{62}$ M. Paniagua, A. Aguado, M. Lara, and O. Roncero, J. Chem. Phys. 109, 2971 (1998).
${ }^{63}$ M. Paniagua, A. Aguado, M. Lara, and O. Roncero, J. Chem. Phys. 111, 6712 (1999)

${ }^{64}$ J. C. Polanyi and W. H. Wong, J. Chem. Phys. 51, 1439 (1969).

${ }^{65}$ M. H. Mok and J. C. Polanyi, J. Chem. Phys. 51, 1451 (1969).

${ }^{66}$ D. R. Herschbach, Adv. Chem. Phys. 10, 319 (1966).

${ }^{67}$ J.-M. Mestdagh, B. Soep, M.-A. Gaveu, and J.-P. Visitcot, Int. Rev. Phys. Chem. 22, 285 (2003).

${ }^{68}$ F. J. Aoiz, M. T. Martínez, M. Menéndez, V. Sáez Rábanos, and E. Verdasco, Chem. Phys. Lett. 299, 25 (1999)

${ }^{69}$ S. Gómez-Carrasco, L. González-Sánchez, A. Aguado, O. Roncero, J. M. Alvariño, M. L. Hernández, and M. Paniagua, J. Chem. Phys. 121, 4605 (2004).

${ }^{70}$ L. González-Sánchez, S. Gómez-Carrasco, A. Aguado, M. Paniagua, M. L. Hernández, J. M. Alvariño, and O. Roncero, J. Chem. Phys. 121, 309 (2004).

${ }^{71}$ T. Xie, J. Bowman, J. W. Duff, M. Braunstein, and B. Ramachandran, J. Chem. Phys. 122, 014301 (2005).

${ }^{72}$ T. Xie, D. Wang, J. M. Bowman, and D. E. Manolopoulos, J. Chem. Phys. 116, 7461 (2002).

${ }^{73}$ O. I. Tolstikhin and H. Nakamura, J. Chem. Phys. 108, 8899 (1998).

${ }^{74}$ K. Nobusada, H. Nakamura, Y. Lin, and B. Ramachandran, J. Chem. Phys. 113, 1018 (2000).

${ }^{75}$ A. Dupays, A. Beswick, B. Lepetit, C. Rizzo, and D. Bakalov, Phys. Rev. A 68, 062506 (2003).

${ }^{76}$ A. Dupays, A. Beswick, B. Lepetit, C. Rizzo, and D. Bakalov, Phys. Rev. A 69, 062501 (2004)

${ }^{77}$ H. Kamisaka, O. I. Tolstikhin, and H. Nakamura, J. Phys. Chem. A 108, 8827 (2004)

${ }^{78}$ B. Lepetit and A. Dypays (private communication).

${ }^{79}$ A. Lichnerowicz, Elementos de Cálculo Tensorial (Aguilar, Madrid, 1962). 